\title{
THE GENERA OF AMALGAMATIONS OF GRAPHS $\left({ }^{1}\right)$
}

\author{
BY
}

SETH R. ALPERT

ABSTRACT。 If $p \leq m, n$ then $K_{m} \vee_{K_{p}} K_{n}$ is the graph obtained by identifying a copy of $K_{p}$ contained in $K_{m}$ with a copy of $K_{p}$ contained in $K_{n}$. It is shown that for all integers $p \leq m, n$ the genus $g\left(K_{m} \vee_{K_{p}} K_{n}\right)$ of $K_{m} \vee_{K_{p}} K_{n}$ is less than or equal to $g\left(K_{m}\right)+g\left(K_{n}\right)$. Combining this fact with the lower bound obtained from the Euler formula, one sees that for $2 \leq p \leq 5, g\left(K_{m} \vee_{K_{p}} K_{n}\right)$ is either $g\left(K_{m}\right)+g\left(K_{n}\right)$ or else $g\left(K_{m}\right)+g\left(K_{n}\right)-1$. Except in a few special cases, it is determined which of the se values is actually attained.

\section{PRELIMINARIES AND SUMMARY OF RESULTS}

A. Background. For the purposes of this discussion, a graph is a finite simplicial 1-complex. In particular, graphs have finitely many vertices, no loops, and no multiple edges. Unless otherwise specified, the graphs considered here are assumed to be connected.

The genus of a graph $G$ is the minimum genus $g(G)$ of any closed orientable 2 -manifold in which $G$ can be imbedded. A classical problem in graph theory is to find a method for computing the genus of an arbitrary graph. Elegant algorithms due to Edmonds [3] (for details see Youngs [17]) and Boland [2] solve this problem theoretically, but both are too lengthy to be of practical computational use.

Much of the progress in this area has been in the computation of the genera of certain special classes of graphs. The most important result of this type is the determination of the genera of the complete graphs. Specifically, the complete graph $K_{n}$ has vertices $1,2, \cdots, n$ and an edge between each pair of distinct vertices. For any real number $x$, the ceiling of $x$ is the least integer $\{x\}$ greater than or equal to $x$.

Complete Graph Theorem. For all $n \geq 3$,

$$
g\left(K_{n}\right)=\{(n-3)(n-4) / 12\} .
$$

The long and difficult proof of this theorem is accomplished in twelve cases,

Received by the editors June $19,1972$.

AMS (MOS) subject classifications (1970). Primary 05C10; Secondary 55A15. Theorem.

Key words and phrases. Orientable 2-manifold, genus, Euler formula, Complete Graph

( $\left.{ }^{1}\right)$ This research constitutes the author's doctoral thesis submitted in partial fulfillment of the requirements for the degree of Doctor of Philosophy at Columbia University. The author wishes to thank Professors Hyman Bass and Jonathan Gross for their guidance and advice during the course of this research. 
depending on the residue class of $n$ modulo 12 [10]. It is due to Ringel and Youngs with the aid of Gustin, Mayer, Terry, and Welch ([4], [7], [8], [9], [10], [11], [12], [13], [14], [15], [18], [19]).

A graph $G$ will be called critical if for each edge $e$ of $G, g(G \sim e)=g(G)-1$. The following theorem on critical complete graphs is obtained as part of the proof of the Complete Graph Theorem.

Theorem I.1. If $m=12 s+5$ and $s \geq 0$ or if $m=12 s+2$ and $s \geq 1$ is odd, then $K_{m}$ is critical.

B. Statement of the problem. An approach to the problem of genus suggested by Harary is to consider the genera of amalgamations of graphs. Specifically, suppose $T$ is a subgraph both of $G$ and of $H$. Fix a copy of $T$ contained in $G$ and another contained in $H$. The amalgamation of $G$ and $H$ along $T$ is the graph $G \vee_{T} H$ obtained by identifying the fixed copies of $T$, consolidating any multiple edges which may arise. Of course, this terminology is imprecise because both $G$ and $H$ may contain several different copies of $T$. However, the main interest here is in the case when $G$ and $H$ are complete, and therefore $G \vee_{T} H$ is completely determined by the isomorphism class of $T$. Thus no confusion will arise from the use of this terminology in the present context.

One might hope to find some relationship between the genus of $G \vee_{T} H$ and the genera of $G, H$, and $T$. In fact, when $T$ consists of a single vertex, Battle, Harary, Kodama, and Youngs [1] have obtained the following result.

Theorem I.2. For all graphs $G$ and $H$,

$$
g\left(G \vee_{K_{1}} H\right)=g(G)+g(H) .
$$

A simple example demonstrates that no such relationship need hold when $T$ is more complicated. Let $G, H$, and $T$ be as in Figure 1 .
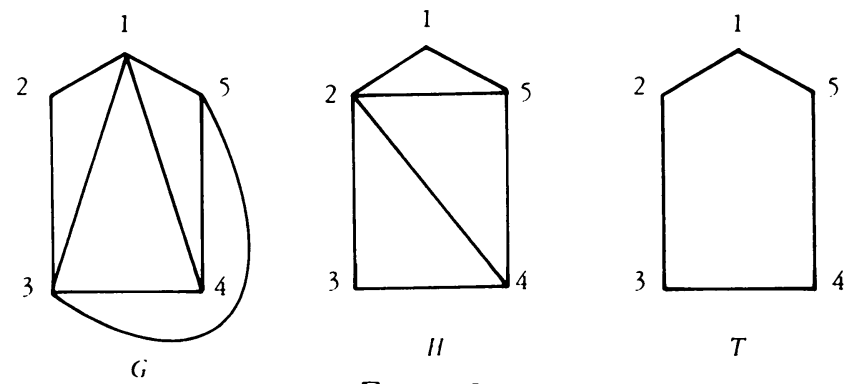

Figure 1

Clearly $G, H$, and $T$ are of genus 0 and $G \vee_{T} H=K_{5}$. It is well known that $g\left(K_{5}\right)=1$.

Now let $G_{n}, H_{n}$, and $T_{n}$ be graphs obtained by amalgamating $n$ copies of $G$, 
$H$, and $T$, respectively, along the single vertex 1 . It follows from Theorem I.2 and from induction that $g\left(G_{n} \vee T_{n} H_{n}\right)=n$ and that $G_{n}, H_{n}$, and $T_{n}$ are all of genus 0 .

In order to avoid such difficulties, attention here will be focused on amalgamations of complete graphs.

C. Summary of results. The major results obtained in this paper are for amalgamations of complete graphs along $K_{p}, p \leq 5$, and are summarized in the following theorems.

The following upper bound for $g\left(K_{m} \vee K_{p} K_{n}\right)$ will be obtained as Proposition I.15 in $\S \mathrm{I}$. E.

Theorem I.3. For all $m, n$, and $p$ such that $m$ and $n \geq p$,

$$
g\left(K_{m} \vee_{K_{p}} K_{n}\right) \leq g\left(K_{m}\right)+g\left(K_{n}\right) .
$$

When one or both of $K_{m}$ and $K_{n}$ is critical and $p$ is sufficiently large, this upper bound can be decreased by 1 or 2 as it is shown in $\$$ III.D. For large $p$ this is probably a poor upper bound, but improving on it seems to be quite difficult. Further comments on this appear in $\$$ IV.C.

The following corollary to Theorem I. 3 is obtained in $\S$ I.E.

Theorem 1.4. If $p \leq 5$, then for all $m$ and $n \geq p, g\left(K_{m} \vee K_{p} K_{n}\right)$ is either $g\left(K_{m}\right)+$ $g\left(K_{n}\right)$ or $g\left(K_{m}\right)+g\left(K_{n}\right)-1$.

Chapter II is devoted to proving the following result.

Theorem 1.5 ( $K_{2}$-amalgamations). (1) If $g\left(K_{m} \vee_{K_{2}} K_{n}\right)=g\left(K_{m}\right)+g\left(K_{n}\right)-1$, then $m$ is congruent to 2 or 5 modulo 12, and $n$ is congruent to 2 or 5 modulo 12 .

(2) If $K_{m}$ and $K_{n}$ are critical, then $g\left(K_{m} \vee K_{2} K_{n}\right)=g\left(K_{m}\right)+g\left(K_{n}\right)-1$.

Note that, in conjunction with Theorems I.1 and I.4, Theorem I.5 gives a determination of $g\left(K_{m} \vee K_{2} K_{n}\right)$ for all $m$ and $n$ except when one of $m$ and $n$ is congruent to 2 or 5 modulo 12 and the other is of the form $24 s+2, s \geq 1$.

This gap would be filled if it could be shown that all graphs of the form $K_{24 s+2}$ were critical. Because this problem remains unsolved, there are similar gaps in the following theorems, all of which are proven in Chapter III.

Theorem I.6 ( $K_{3}$-amalgamations). (1) 'If $g\left(K_{m} \vee K_{3} K_{n}\right)=g\left(K_{m}\right)+g\left(K_{n}\right)-1$, then one of $m$ and $n$ is congruent to 2 or 5 modulo 12, and the other is congruent to $1,2,5,6,9$, or 10 modulo 12 .

(2) If $K_{m}$ and $K_{n}$ are critical, then $g\left(K_{m} \vee K_{3} K_{n}\right)=g\left(K_{m}\right)+g\left(K_{n}\right)-1$.

(3) If $K_{m}$ is critical and $n$ is congruent to $1,6,9$, or 10 modulo 12, then $g\left(K_{m} \vee K_{3} K_{n}\right)=g\left(K_{m}\right)+g\left(K_{n}\right)-1$ if and only if $K_{n}$ bas a minimal imbedding with a 6-sided face which bas a repeated vertex (these terms are defined in the next 
section). By symmetry, the same statement with the roles of $m$ and $n$ interchanged is also true.

Theorem I.7 ( $K_{4}$-amalgamations). (1) If $g\left(K_{m} \vee K_{4} K_{n}\right)=g\left(K_{m}\right)+g\left(K_{n}\right)-1$, then one of $m$ and $n$ is congruent to 2 or 5 modulo 12 and the other is congruent to $1,2,5,6,8,9,10$, or 11 modulo 12 .

(2) If $K_{m}$ and $K_{n}$ are critical, then $g\left(K_{m} \vee_{K_{4}} K_{n}\right)=g\left(K_{m}\right)+g\left(K_{n}\right)-1$.

(3) If one of $m$ and $n$ is congruent to 5 modulo 12 and the other is congruent to $1,2,5,6,8,9,10$, or 11 modulo 12 , then $g\left(K_{m} \vee K_{4} K_{n}\right)=g\left(K_{m}\right)+g\left(K_{n}\right)-1$.

Theorem I.8 ( $K_{5}$-amalgamations). (1) If $g\left(K_{m} \vee K_{5} K_{n}\right)=g\left(K_{m}\right)+g\left(K_{n}\right)-1$, then one of the following conditions is true.

(a) One of $m$ and $n$ is congruent to 2 or 5 modulo 12.

(b) One of $m$ and $n$ is congruent to 8 or 11 modulo 12 and the other is congruent to $1,6,8,9,10$, or 11 modulo 12 .

(2) If $K_{m}$ and $K_{n}$ are critical, then $g\left(K_{m} \vee K_{5} K_{n}\right)=g\left(K_{m}\right)+g\left(K_{n}\right)-1$.

(3) If one of $m$ and $n$ is congruent to 5 modulo 12, then $g\left(K_{m} \vee K_{5} K_{n}\right)=$ $g\left(K_{m}\right)+g\left(K_{n}\right)-1$.

Note that an immediate consequence of the parts (2) of the above theorems is the following.

Corollary. If $K_{m}$ and $K_{n}$ are critical and $2 \leq p \leq 5$, then $g\left(K_{m} \vee K_{p} K_{n}\right)=$ $g\left(K_{m}\right)+g\left(K_{n}\right)-1$.

Some additional results of this type appear in $\$$ III.D.

For an explanation of the congruence conditions on $m$ and $n$ of the above theorems, see the remark following Table I.1.

D. Basic properties of imbeddings of graphs. This section is devoted to developing some terminology and basic facts about imbeddings of graphs in closed orientable surfaces. To avoid trivialities, assume that graphs other than $K_{2}$ do not have points of degree one.

If $f: G \rightarrow M$ is an imbedding of a graph in an orientable surface, the image of $f$ is also called $G$.

The components of $M \sim G$ are called faces.

If all faces are open 2-cells, the imbedding is a 2-cell imbedding.

A standard topological cutting argument shows that any minimal imbedding is a 2-cell imbedding. Youngs [17] gives such an argument in detail.

A 2-cell imbedding of $G$ in $M$ gives a cellular decomposition of $M$ into the faces. Hence the well-known Euler formula holds. In particular, if $V(G)$ is the number of vertices of a graph $G$ and $E(G)$ is the number of edges, then for any 
2-cell imbedding of $G$ in a surface of genus $g$ with $F$ faces the Euler formula is

$$
2-2 g=V(G)-E(G)+F .
$$

Note that the boundary of any face $P$ of a 2 -cell imbedding of $G$ is a closed walk of $G$. If the length of this walk is $n$, then $P$ is called an $n$-sided face.

The orientation on a surface $M$ induces an orientation on the boundary of any face of a 2-cell imbedding of a graph in $M$. A convention to be followed here is to give the boundary of a face of a 2-cell imbedding the opposite orientation. The purpose of this is notational and will be clarified in $\$$ I.F on schemes.

Using this orientation, the boundary of any face of a 2-cell imbedding of a graph $G$ may now be thought of as a directed closed walk of $G$. The face itself will be referred to and denoted by the cyclic sequence of vertices of its boundary, enclosed by square brackets. Except where some confusion might arise, no attempt will be made to distinguish between faces of 2-cell imbeddings and their boundaries.

This is illustrated by Figure 2, where the graph $G$ is imbedded in the 2-sphere. There, and throughout this paper unless otherwise specified, the orientation is taken to be counterclockwise. The displayed imbedding has faces $[1,4,2],[2,4$, $3]$, and $[1,2,3,4]$.

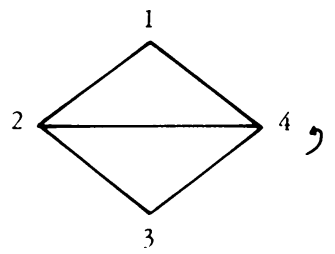

Figure 2

An $n$-sided face $P$ of a 2 -cell imbedding may very well have fewer than $n$ distinct vertices or edges on its boundary. A vertex or edge appearing more than once on $P$ will be called a repeated vertex or edge of $P$. Because graphs do not have multiple edges, $P$ can have a repeated vertex only if $n \geq 6$ and a repeated edge only if $n \geq 8$. Faces without repeated vertices or edges will often be referred to as polygons and given the usual geometric names. Thus all 3-sided faces are triangles, all 4-sided faces are quadrilaterals, and all 5-sided faces are pentagons.

If $x$ is a vertex of a graph imbedded in a surface $M$, the orientation on $M$ induces a cyclic ordering of the vertices adjacent to $x$ which will be called the adjacency tour at $x$. This ordering is obtained by taking a small loop about $x$ and looking at the intersections of the loop with the edges emanating from $x$ in the order given by the induced orientation on the loop.

Given a 2 -cell imbedding of $G$ in $M$, the adjacency tour at a vertex $x$ of $G$ will be denoted by writing $x$ ) followed by the adjacencies to $x$ written in the proper 
cyclic order. For example, the adjacency tours at the vertices of the imbedding displayed in Figure 2 are 1) 2, 4, 2) 3, 4, 1, 3) 4, 2, and 4) 1, 2, 3.

An angle of a face $P$ of a 2 -cell imbedding is an ordered triple of vertices, written $a b c$, such that $P=[a, b, c, \cdots]$. The vertex $b$ is the center of the angle and $a$ and $c$ are its endpoints. Clearly $a b c$ is an angle of some face of a 2-cell imbedding if and only if $c$ is the successor of $a$ in the adjacency tour at $b$.

These concepts will be further elaborated upon in $\S$ I.F.

The triangulation deficiency $d(G, g)$ of a graph $G$ for genus $g$ is defined to be

$$
d(G, g)=6 g-6+3 V(G)-E(G) \text {. }
$$

In case $g=g(G), d(G, g(G))$ is simply called the triangulation deficiency of $G$ and denoted $d(G)$.

If there is a 2-cell imbedding of $G$ in a surface of genus $g$ with $F$ faces, then an elementary manipulation of the Euler formula shows

$$
d(G, g)=6 g-6+3 V(G)-E(G)=2 E(G)-3 F .
$$

The motivation for this terminology is made clear by the following proposition. Assume that $G$ has no vertices of degree one.

Proposition I.9. (1) If there is a 2-cell imbedding of $G$ in a surface of genus $g$ and if $F_{k}$ is the number of k-sided faces, then

$$
d(G, g)=\sum_{k \geq 3}(k-3) F_{k} .
$$

(2) If $d(G, g) \geq 0$, then it is the number of edges which would have to be added to a 2-cell imbedding of $G$ in a surface $M$ of genus $g$ in order to make it a triangulation of $M$.

(3) If $d(G, g)<0, G$ cannot be imbedded in a surface of genus $g$, and at least $|d(G, g)|$ edges would have to be removed from $G$ to make such an imbedding possible (even though that might not always work).

Proof. (1) In a 2-cell imbedding of $G$ each edge appears twice as the side of a face. Hence $2 E(G)=\Sigma_{k} F_{k}$. Moreover, $G$ has no loops or multiple edges, so this sum may be taken over all $k \geq 3$. It follows that

$$
d(G, g)=2 E(G)-3 F=\sum_{k \geq 3} k F_{k}-3 \sum_{k \geq 3} F_{k}=\sum_{k \geq 3}(k-3) F_{k} .
$$

(2) By (1), if $d(G, g)>0$ there is at least one nontriangular face in any 2cell imbedding of $G$ in a surface of genus $g$. This means that an edge (possibly multiple) can be drawn across that face, increasing $E(G)$ and $F$ each by one, and hence decreasing $2 E(G)-3 F$ by one. The conclusion now follows by iteration of this procedure. Of course, $d(G, g) \geq 0$ does not guarantee the existence of any 
imbeddings of $G$ in a surface of genus $g$.

(3) By (1), if $d(G, g)<0$ there is no 2-cell imbedding of $G$ in a surface of genus $g$ and hence no imbedding at all. If $|d(G, g)|$ edges are removed from $G$ to yield a new graph $G^{\prime}$, then by definition of triangulation deficiency, $d\left(G^{\prime}, g\right)=0$. Hence an imbedding of $G^{\prime}$ in a surface of genus $g$ could possibly exist.

Corollary I.10. For any graph $G$ without vertices of degree 1,

$$
g(G) \geq\{E(G) / 6-V(G) / 2+1\} .
$$

Proof. A minimal imbedding is a 2 -ceil imbedding and so $d(G) \geq 0$. That is,

$$
6 g(G)-6+3 V(G)-E(G) \geq 0,
$$

from which the conclusion readily follows.

E. Bounds on $g\left(K_{m} \vee_{K_{p}} K_{n}\right)$-Proof of Theorems I.3 and I.4.

Proposition I.11. For all graphs $G, H$, and $T$,

$$
d\left(G \bigvee_{T} H, g(G)+g(H)-g(T)+x\right)=d(G)+d(H)-d(T)+G x .
$$

Hence $g(G \vee T H) \geq g(G)+g(H)-g(T)+\{(d(T)-d(G)-d(H)) / 6\}$.

Proof. By definition of triangulation deficiency, for any graph $G, d(G)=$ $6 g(G)-6+3 V(G)-E(G)$.

Observe that $V\left(G \vee_{T} H\right)=V(G)+V(H)-V(T)$ and $E\left(G \vee_{T} H\right)=E(G)+E(H)-$ $E(T)$. Consequently,

$$
\begin{aligned}
&\left.d\left(G \vee_{T} H\right), g(G)+g(H)-g(T)+x\right) \\
&= 6 g(G)+6 g(H)-6 g(T)+6 x-6+3 V\left(G \vee_{T} H\right)-E\left(G \vee_{T} H\right) \\
&= 6 x+6 g(G)-6+3 V(G)-E(G)+6 g(H) \\
&-6+3 V(H)-E(H)-6 g(T)+6-3 V(T)+E(T) \\
&= 6 x+d(G)+d(H)-d(T) .
\end{aligned}
$$

Since $d\left(G \vee_{T} H\right) \geq 0$ and since $g\left(G \vee_{T} H\right)=g(G)+g(H)-g(T)+x$ for some integer $x$, it follows that $6 x+d(G)+d(H)-d(T) \geq 0$ and so

$$
x \geq\{(d(T)-d(G)-d(G)) / 6\} .
$$

Hence

$$
g\left(G \vee_{T} H\right) \geq g(G)+g(H)-g(T)+\{(d(T)-d(G)-d(H)) / 6\}
$$

Proposition I.12. For all integers $q \geq 3$,

$$
d\left(K_{q}\right)=6(\{(q-3)(q-4) / 12\}-(q-3)(q-4) / 12) .
$$

Proof. By the Complete Graph Theorem, $g\left(K_{q}\right)=\{(q-3)(q-4) / 12\}$. Since $K_{q}$ has $q$ vertices and $\left(q^{2}-q\right) / 2$ edges, 


$$
\begin{aligned}
d\left(K_{q}\right) & =6\{(q-3)(q-4) / 12\}-6+3 q-\left(q^{2}-q\right) / 2 \\
& =6\{(q-3)(q-4) / 12\}-\left(q^{2}-7 q+12\right) / 2 \\
& =6(\{(q-3)(q-4) / 12\}-(q-3)(q-4) / 12) .
\end{aligned}
$$

A consequence of Proposition $\mathrm{I} .12$ is that the triangulation deficiency of $K_{q}$ depends only on the residue class of $q$ modulo 12 . For example, if $q$ is congruent to 5 modulo 12 , then $q=12 s+5$ for some $s$, and

$$
d\left(K_{12 s+5}\right)=6(\{(12 s+2)(12 s+1) / 12\}-(12 s+2)(12 s+1) / 12)=5 .
$$

In Table I.1, the triangulation deficiency of $K_{q}$ for all $q \geq 3$ is given as a function of the residue class of $q$ modulo 12 . Note that the only possible values for $d\left(K_{q}\right)$ are $0,2,3$, and 5 .

\section{Table I.1}

$d\left(K_{q}\right)$ as a function of the residue class of $q$ modulo $12, q \geq 3$

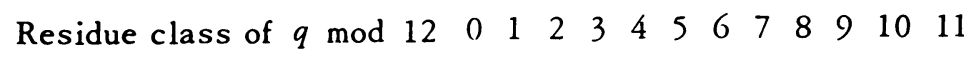

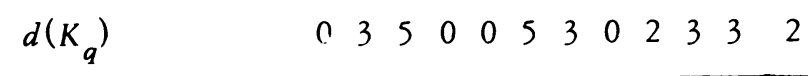

Comparing Table I. 1 to the congruence requirements on $m$ and $n$ in Theorems I.5-I.8, one sees that most often the restrictions amount to asking that $d\left(K_{q}\right)$ assume one or more of the four possible values $0,2,3$, and 5 . In fact

$$
\begin{array}{ll}
n \equiv 0,3,4, \text { or } 7 \bmod 12 & \text { if and only if } d\left(K_{n}\right)=0 \\
n \equiv 8 \text { or } 11 \bmod 12 & \text { if and only if } d\left(K_{n}\right)=2, \\
n \equiv 1,6,9, \text { or } 10 \bmod 12 & \text { if and only if } d\left(K_{n}\right)=3, \\
n \equiv 2 \text { or } 5 \bmod 12 & \text { if and only if } d\left(K_{n}\right)=5 .
\end{array}
$$

Corollary I.13. If $K_{q}$ is critical, then $q$ is congruent to 2 or 5 modulo 12 and there is a triangular imbedding of $K_{q} \sim K_{2}$.

Proof. Suppose $K_{q}$ is critical. Then $K_{q} \sim K_{2}$ can be imbedded in a surface of genus $g\left(K_{q}\right)-1$. Hence $d\left(K_{q} \sim K_{2}, g\left(K_{q}\right)-1\right) \geq 0$. But by definition of triangulation deficiency, $d\left(K_{q} \sim K_{2}, g\left(K_{q}\right)-1\right)=d\left(K_{q}\right)-5$.

Hence $d\left(K_{q}\right) \geq 5$. The result now follows from Proposition I.12 (see Table I.1), since $d\left(K_{q} \sim K_{2}, g\left(K_{q}\right)-1\right)$ must equal 0 , and therefore any imbedding has only triangular faces.

Corollary I.14. If $2 \leq p \leq 5$, then for all $m$ and $n$,

$$
g\left(K_{m} \vee_{K_{p}} K_{n}\right) \geq g\left(K_{m}\right)+g\left(K_{n}\right)-1 .
$$


Proof. If $2 \leq p \leq 4$, then $d\left(K_{p}\right)$ is either 0 or -1 . Hence $d\left(K_{p}\right)-d\left(K_{m}\right)-$ $d\left(K_{n}\right) \geq-11$ and by Proposition I.11,

$$
\begin{aligned}
g\left(K_{m} \vee_{K_{p}} K_{n}\right) & \geq g\left(K_{m}\right)+g\left(K_{n}\right)-g\left(K_{p}\right)+\left\{\left(d\left(K_{p}\right)-d\left(K_{m}\right)-d\left(K_{n}\right)\right) / 6\right\} \\
& \geq g\left(K_{m}\right)+g\left(K_{n}\right)-1 .
\end{aligned}
$$

If $p=5$, then $d\left(K_{5}\right)=5$ and $g\left(K_{5}\right)=1$, so $d\left(K_{p}\right)-d\left(K_{m}\right)-d\left(K_{n}\right) \geq-5$. The conclusion follows from Proposition I.11.

If graphs $G$ and $H$ are amalgamated along an edge $e$, it is easy to see that $g\left(G \vee_{e} H\right) \leq g(G)+g(H)$. For if $G$ and $H$ are minimally imbedded in surfaces $M_{1}$ and $M_{2}$, respectively, then $e$ must lie on the boundary of a face $P_{1}$ of $M_{1}$ and a face $P_{2}$ of $M_{2}$. Since the imbeddings of $G$ and $H$ are minimal, $P_{1}$ and $P_{2}$ are open 2-cells and hence it is possible to excise from them open 2-cells $C_{1}$ and $C_{2}$ such that the boundary of $C_{i}$ intersects the boundary of $P_{i}$ precisely in $e$, as in Figure 3.

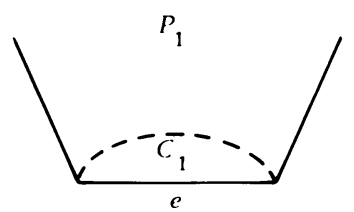

Local picture on $M_{1}$

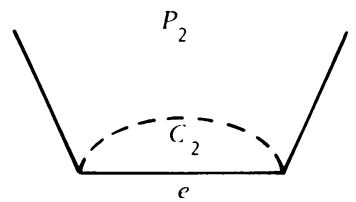

Local picture on $M_{2}$

Figure 3

If the resulting boundaries are identified so that the copies of $e$ match up, the result is an imbedding of $G \vee_{\boldsymbol{e}} H$ in the connected sum of $M_{1}$ and $M_{2}$, that is, in a surface of genus $g(G)+g(H)$.

Suppose that complete graphs $K_{m}$ and $K_{n}$ are amalgamated along $K_{3}$. Note that $K_{m} \vee_{K_{3}} K_{n}$ is independent of which triangular subgraphs of $K_{m}$ and $K_{n}$ one amalgamates along. Any minimal imbedding of $K_{m}, m \geq 3$, has several triangular faces. Hence one can select triangular faces in minimal imbeddings of $K_{m}$ and $K_{n}$, excise them from the respective surfaces, and identify the resulting boundaries in such a way so that vertices and edges match up to obtain an imbedding of $K_{m} \vee K_{3} K_{n}$ in a surface of genus $g\left(K_{m}\right)+g\left(K_{n}\right)$. It follows that $g\left(K_{m} \vee K_{3} K_{n}\right) \leq$ $g\left(K_{m}\right)+g\left(K_{n}\right)$.

These ideas can be extended to amalgamations of complete graphs along any $K_{p}$, as it will be shown in the following proposition, originally stated as Theorem I.3.

Proposition 1.15. For all $m, n$, and $p, g\left(K_{m} \vee K_{p} K_{n}\right) \leq g\left(K_{m}\right)+g\left(K_{n}\right)$.

Proof. If $p=1$, apply Theorem I.2. For $p=2$ the preceding argument gives the desired result. So assume $p \geq 3$. 
Given a minimal imbedding of $K_{m}$ in a surface $M$ select a vertex 1 with adjacency tour 1) $2,3, \cdots, m$.

For all $i$ such that $2 \leq i \leq p-1, i 1(i+1)$ is an angle of some face of the imbedding. Because $K_{m}$ is complete there exists an edge between $i$ and $i+1$. Hence if $i 1(i+1)$ is not already an angle of a triangular face, the edge $(i, i+1)$ may be moved from its original location in the imbedding so as to make the triangle complete. There is therefore no loss in generality in assuming that for all $i$ such that $2 \leq i \leq p-1$, the angle $i 1(i+1)$ is an angle of a triangular face.

Now delete all edges between 1 and $3,4, \cdots, p-1$ to obtain an imbedding of $K_{m} \sim K_{1}, p-3$ in a surface of genus $g\left(K_{m}\right)$ with a $p$-sided face $P=[p, p-1$, $\cdots, 2,1]$. This is illustrated in Figure $4 a$.

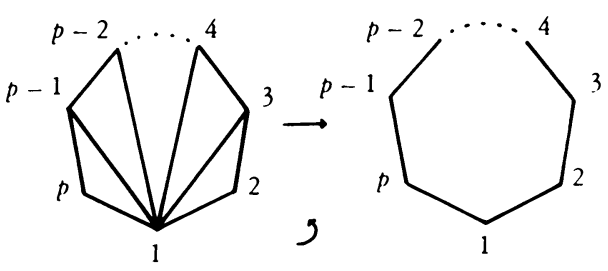

a

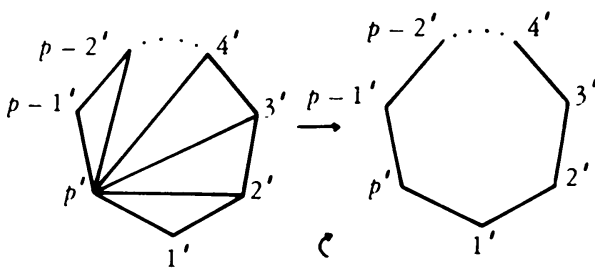

b

Figure 4

Similarly, given a minimal imbedding of $K_{n}$ in a surface $M^{\prime}$, select a vertex $p^{\prime}$ with adjacency tour

$$
\left.p^{\prime}\right) n^{\prime}, n-1^{\prime}, \ldots,(p+1)^{\prime},(p-1)^{\prime}, \ldots, 2^{\prime}, 1^{\prime} .
$$

As before, assume that all angles $(i+1)^{\prime} p^{\prime}{ }^{\prime}{ }^{\prime}$ with $1 \leq i \leq p-2$ are angles of triangular faces. As is illustrated in Figure $4 b$, where $M^{\prime}$ is given a clockwise orientation, one can now delete all edges between $p^{\prime}$ and $2^{\prime}, 3^{\prime}, \cdots,(p-2)^{\prime}$ to obtain an imbedding of $K_{n} \sim K_{1}, p-3$ in a surface of genus $g\left(K_{n}\right)$ with a $p$-sided face $P^{\prime}=\left[1^{\prime}, 2^{\prime}, \cdots, p^{\prime}\right]$.

Excise $P$ from $M$ and $P^{\prime}$ from $M^{\prime}$ and identify the boundaries so that the vertex $i$ of $K_{m}$ is matched up with the vertex $i^{\prime}$ of $K_{n}, 1 \leq i \leq p$, and so that the corresponding edges match up. Note that this identification is made so that the resulting surface is still orientable.

After deleting any multiple edges which arise from the identification, one obtains an imbedding of a graph $G$ in the connected sum of $M$ and $M^{\prime}$, that is, in a surface of genus $g\left(K_{m}\right)+g\left(K_{n}\right)$. Since $K_{m}$ and $K_{n}$ are complete, it is clear that the edges deleted from them to form the polygons $P$ and $P^{\prime}$ are restored by the identification. Hence $G=K_{m} \vee K_{p} K_{n}$ and the proof is complete.

Notes. (1) In certain special cases, the type of construction used in the proof of Proposition I.15 can be made for lower genus subgraphs of $K_{m}$ and $K_{n}$ in such a way so that the identification process restores enough missing edges 
to yield an imbedding of $K_{m} \vee_{K_{p}} K_{n}$ in a surface of genus less than $g\left(K_{m}\right)+g\left(K_{n}\right)$. For examples, see $\S$ III.D.

(2) Observe that $K_{m} \vee_{K_{p}} K_{n}=K_{m+n-p} \sim K_{m-p, n-p}$. Thus an obvious upper bound for $g\left(K_{m} \vee_{K_{p}} K_{n}\right)$ is $g\left(K_{m+n-p}\right)$. However, this is an improvement on the bound obtained in Proposition I.15 only when $p$ is close to $m$ or $n$.

Corollary (Theorem I.4). If $p \leq 5$, then for all $m$ and $n, g\left(K_{m} \vee_{K_{p}} K_{n}\right)$ is either $g\left(K_{m}\right)+g\left(K_{n}\right)$ or $g\left(K_{m}\right)+g\left(K_{n}\right)-1$.

Proof. This is an immediate consequence of Corollary I.14 and Proposition I.15.

F. Schemes. When studying imbeddings of graphs in surfaces of higher genus, it soon becomes apparent that trying to draw pictures is generally insufficient. What is needed is some simple means of describing an imbedding of a graph. Actually, this is at hand in some of the concepts developed in \$I.D.

A scheme for a 2-cell imbedding of a graph is a list of the adjacency tours at each vertex as given by the imbedding. Clearly, any 2 -cell imbedding gives rise to a scheme.

Conversely, given a graph $G$, one can arbitrarily prescribe an "adjacency tour" at a vertex $x$ of $G$ by writing in any cyclic order those vertices adjacent to $x$. A result of Edmonds [3], given in detail by Youngs [17], states that a list of such "adjacency tours," one for each vertex of $G$, actually is a scheme for a 2 cell imbedding of $G$ in some orientable surface.

Youngs gives an explicit process for constructing the imbedding associated to such a scheme. Because of its frequent use in what follows, it will now be discussed in some detail.

Let $G$ be a graph with vertices $1,2, \cdots, n$ and suppose that a scheme for $G$ is given. For each $i$, define a cyclic permutation $P_{i}$ of the vertices adjacent to $i$ by sending a vertex to its immediate successor in the adjacency tour at $i$.

The permutations $P_{1}, \cdots, P_{n}$ define a permutation $P$ on the set of directed edges of $G$ given by $P(x, y)=\left(y, P_{y}(x)\right)$. Because $x$ is adjacent to $y, P$ is well defined.

Let $R$ be an orbit of $P$ of length $k$, and let $S$ be an oriented 2-cell in the form of a polygon with $k$ sides. Pick a directed edge $(a, b)$ in $R$ and label any side of $S(a, b)$. The next side of $S$ in the chosen direction of the orientation is labeled $P(a, b)$, and so on until the successive sides of $S$ in the direction of the orientation have been named

$$
(a, b), P(a, b), P^{2}(a, b), \ldots, P^{k-1}(a, b) .
$$

If $R_{1}, \cdots, R_{F}$ is the collection of orbits of $P$, the process described above results in oriented 2-cells $S_{1}, \cdots, S_{F}$ with sides labeled by the directed edges 
of $G$. This collection has the property that if $(a, b)$ is the side of one of $S_{1}, \cdots$, $S_{F}$, then $(b, a)$ also appears as a side of one of them and each appears exactly one time in this way. Because $G$ is connected, no nonempty proper subset of $S_{1}$, $\cdots, s_{F}$ has this property.

Hence the usual identification procedure of combinatorial topology produces an orientable 2-manifold $M$. By definition of $P$, and since each directed edge of $G$ lies in some orbit, the sides of the 2-cells $s_{1}, \cdots, s_{F}$ on $M$ constitute a 2 -cell imbedding of $G$ in $M$. The orientations on $S_{1}, \cdots, S_{F}$ induce an orientation on $M$. The opposite orientation on $M$ yields an adjacency tour at $i$ such that the successor to a vertex $k$ is $P_{i}(k)$, for all $i$. The convention here will be to use this opposite orientation on $M$.

It should be mentioned that this construction yields an algorithm for computing the genus of an arbitrary graph. The genus of the imbedding associated to a scheme is found by computing the number of orbits, that is, faces, and applying Euler's formula. The minimum among all genera of schemes for a graph is the genus of the graph. Since the number of schemes for a graph with vertices $v_{1}$, $v_{2}, \cdots, v_{n}$ is $\Pi_{i=1}^{n}\left(\operatorname{deg}\left(v_{i}\right)-1\right) !$, this algorithm is of no practical use.

An example will give the reader a better feeling for these concepts. Consider the graph displayed in Figure 2, which is reproduced here.

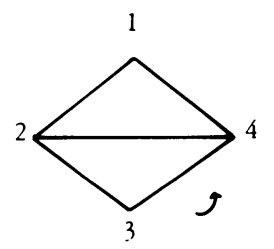

Because vertices 1 and 3 are of degree 2, and by symmetry the only schemes inequivalent under graph automorphism for this graph are
(a) 1) 2, 4
(b) 1) 2, 4
2) $1,3,4$
2) $1,4,3$
3) 2,4
3) 2,4
4) $1,2,3$
4) $1,2,3$.

The required computation shows that scheme (a) has faces $[2,1,4],[1,2,3$, $4]$, and $[2,3,4]$, just as in the figure. The only face of scheme (b) is found to be $[2,1,4,2,3,4,1,2,4,3]$, and so this scheme gives a 2 -cell imbedding of the given graph in the torus.

Some simple relationships between schemes and triangular imbeddings will be used frequently and are therefore worth stating explicitly.

Proposition 1.16 (Ringel [8]). A scbeme for a graph $G$ yields a triangular imbedding of $G$ if and only if one of the following equivalent rules bolds for all 
vertices $i, j, k$ and $l$ of $G$.

$\left(\mathrm{R}^{*}\right)$ If the adjacency tour at $i$ is $\left.i\right) j, k, l, \cdots$, then the adjacency tour at $k$ is $k) l, i, j, \cdots$.

(R1) If the adjacency tour at $i$ is i) $j, k, \cdots$, then the adjacency tour at $k$ is k) $i, j, \cdots$.

(R2) If the adjacency tour at $i$ is i) $k, l, \cdots$, then the adjacency tour at $k$ is k) $l, i, \cdots$.

Proof. If the scheme is for a triangular imbedding of $G$ and if $j, k$, and $l$ are successive vertices in the adjacency tour at $i$, then $j i k$ and $k i l$ are angles of adjacent triangles, as in Figure 5.

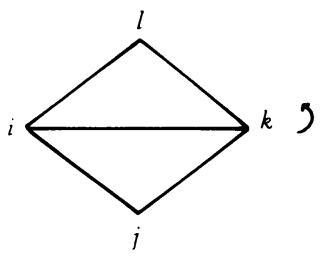

Figure 5

This makes it clear that the adjacency tour at $k$ is $k$ ) $l, i, j, \cdots$, so $\mathrm{R}^{*}$ holds. Clearly, $\mathrm{R}^{*}$ implies $\mathrm{R} 1$ and $\mathrm{R} 2$.

Conversely, suppose $\mathrm{R}^{*}$ holds, and let $P$ be the permutation of the directed edges of $G$ given by the scheme. It suffices to show that every orbit of $P$ is of length 3.

Suppose that $P(x, y)=(y, z)$. Then the adjacency tour at $y$ is $y) x, z, \cdots$ and hence by $\mathrm{R}^{*}$ the adjacency tours at $x$ and $z$ are $\left.x\right) z, y, \cdots$ and $z$ ) $y, x, \cdots$. This means that $P(y, z)=(z, x)$ and $P(z, x)=(x, y)$. Thus the orbit containing $(x, y)$, and so all orbits of $P$, are of length 3 .

If $\mathrm{R} 1$ holds, then $i) j, k, l, \cdots$ implies $k$ ) $i, j, \cdots$ and $l) i, k, \cdots$, which in turn implies $k$ ) $l, i, j, \cdots$. Hence $\mathrm{R} 1$ implies $\mathrm{R}^{*}$.

Similarly, if $\mathrm{R} 2$ holds, then $i) j, k, l, \cdots$ implies $k$ ) $l, i, \ldots$ and $j) k, i, \cdots$, which in turn implies $k$ ) $l, i, j, \cdots$. Thus $\mathrm{R} 2$ implies $\mathrm{R}^{*}$. This completes the proof.

As a final remark, notice that if one of the rules of Proposition I.16 holds for some portion of a scheme, then the faces given by that portion of the scheme are triangular, and conversely.

\section{AMALGAMATIONS ALONG $K_{2}$-PROOF OF THEOREM I.5}

A. Restatement of theorem and analysis of cases. The major goal of this chapter is to prove Theorem I.5, which is now restated as Theorem II.1.

Theorem II.1. (1) If $g\left(K_{m} \vee_{K_{2}} K_{n}\right)=g\left(K_{m}\right)+g\left(K_{n}\right)-1$, then $m$ is congruent to 2 or 5 modulo 12, and $n$ is congruent to 2 or 5 modulo 12 . 
(2) If $K_{n}$ and $K_{m}$ are critical, then $g\left(K_{m} \vee_{K_{2}} K_{n}\right)=g\left(K_{m}\right)+g\left(K_{n}\right)-1$.

The proof will be by cases according to the value of the triangulation deficiency of $K_{m} \vee_{K_{2}} K_{n}$ for genus $g\left(K_{m}\right)+g\left(K_{n}\right)-1$. This is computed using the formula of Proposition I.11 and is displayed in Table II.1 as a function of $d\left(K_{m}\right)$ and $d\left(K_{n}\right)$ (see Table I.1).

\section{Table II. 1}

$$
d\left(K_{m} \vee_{K_{2}} K_{n}, g\left(K_{m}\right)+g\left(K_{n}\right)-1\right) \text { as a function of } d\left(K_{m}\right) \text { and } d\left(K_{n}\right)
$$

\begin{tabular}{r|r|r|r|r|}
$d_{d\left(k_{m}\right)}^{d\left(k_{n}\right)}$ & 0 & 2 & 3 & 5 \\
\hline 0 & -5 & -3 & -2 & 0 \\
2 & -3 & -1 & 0 & 2 \\
3 & -2 & 0 & 1 & 3 \\
5 & 0 & 2 & 3 & 5
\end{tabular}

By Proposition I.9, if the triangulation deficiency is negative then an imbedding is impossible. Hence the uniy cases w consider are when $d\left(K_{m} \vee_{K_{2}} K_{n}, g\left(K_{m}\right)+g\left(K_{n}\right)-1\right)$ is either $0,1,2,3$, or 5. \$II.B is aevoted to proving the impossibility of imbeddings in the first four cases. Referring to the comments following Table I.1, one sees that this will prove (1) of Theorem II.1. The proof of (2) appears in $\$$ II.C, where some special imbeddings are constructed in the remaining case. Whenever possible, results are stated for arbitary amalgamations along $K_{2}$.

B. Nonexistence of imbeddings of $K_{m} \vee_{K_{2}} K_{n}$-Proof of (1) of Theorem Il.1.

Notational Conventions. Inruughout the rest of this paper, $G$ and $H$ will be arbitrary graphs. To avoid trivialities, it will be assumed that neither $G$ nor $H$ has any vertices of degree one. The vertices of any graph of the form $G \vee_{K_{p}} H$ are naturally partitioned into the three sets $G \sim K_{p}, H \sim K_{p}$, and $K_{p}$. Vertices of $G \sim K_{p}$ will be denoted $g_{1}, g_{2}$, etc., those of $H \sim K_{p}$ will be denoted $b_{1}, b_{2}$, etc., and those of $K_{p}$ will be denoted $x, y, z, w$, etc. When writing adjacency tours, three dots separating two vertices of the same type will be used to indicate a sequence of vertices of that type. For example if the adjacency tour at $x$ is x) $g_{1}, \cdots, g_{2}, b_{1}, \cdots, b_{2}$ then it consists of a sequence of vertices of $G \sim K_{p}$ followed by a sequence of vertices of $H \sim K_{p}$.

An amalgamating angle of an imbedding of $G \vee_{K_{t}} H$ is one whose center is 
a vertex of $K_{p}$ and for which one endpoint is a vertex of $G \sim K_{p}$ and the other a vertex of $H \sim K_{p}$. For example, if the adjacency tour at $x$ is $\left.x\right) g_{1}, \cdots, g_{2}, b_{1}$, $\cdots, b_{2}$, then $x$ is the center of the amalgamating angles $g_{2} x b_{1}$ and $b_{2} x g_{1}$. Since no vertex of $G \sim K_{p}$ is adjacent to a vertex of $H \sim K_{p}$, it is clear that an amalgamating angle cannot lie on a triangular face.

To prove (1) of Theorem II.1 it must be shown that no graph of the form $K_{m} \vee_{K_{2}} K_{n}$ can be imbedded in a surface of genus $g\left(K_{m}\right)+g\left(K_{n}\right)-1$ if the triangulation deficiency is less than or equal to 3. This is accomplished in two steps, obtaining somewhat more general results.

First, Theorem II.2 will show that no graph of the form $G \vee_{K_{2}}{ }^{H}$ can be imbedded in a surface of genus $g(G)+g(H)-1$ if the triangulation deficiency is less than or equal to 2 .

Second, The orem II.3 will show that if $G \vee_{K_{2}} H$ can be imbedded in a surface of genus $g(G)+g(H)-1$ and if $d\left(G \vee_{K_{2}} H, g(G)+g(H)-1\right)=3$, then both $G$ and $H$ must have minimal imbeddings of a type which would be impossible if they were complete.

Theorem II.2. Suppose that $G \vee_{K_{2}} H$ is imbedded in a surface of genus $g(G)+$ $g(H)-1$.

(1) If $x$ is a vertex of $K_{2}$, then $x$ is the center of at least two amalgamating angles. If $x$ is the center of exactly two amalgamating angles, then these angles must lie on the same face.

(2) $d\left(G \vee_{K_{2}} H, g(G)+g(H)-1\right) \geq 3$.

Proof. Since the immediate interest here is in (2) the proof will be begun by showing that (1) implies (2).

To see this, assume (1) is true and suppose that $G \vee_{K_{2}} H$ is imbedded in a surface of genus $g(G)+g(H)-1$. If $d\left(G \vee_{K_{2}} H, g(G)+g(H)-1\right) \leq 2$, then there are at most 2 nontriangular faces and any face has at most 5 sides and hence no repeated vertices. Because an amalgamating angle must lie on a nontriangular face, this means that $x$ is the center of at most 2 amalgamating angles, and if there are 2 amalgamating angles with center $x$, then they must lie on distinct faces. Since this contradicts (1), it follows that $d\left(G \vee_{K_{2}} H, g(G)+g(H)-1\right) \geq 3$.

To prove (1), note that the vertices adjacent to $x$ include the other vertex $y$ of $K_{2}$ and at least one vertex from each of $G \sim K_{2}$ and $H \sim K_{2}$. Hence in the adjacency tour at $x$, there is at least one juxtaposition of a vertex of $G \sim K_{2}$ with a vertex of $H \sim K_{2}$. In other words, $x$ is the center of at least one amalgamating angle.

If $x$ is the center of exactly one amalgamating angle, then the adjacency tour at $x$ may be assumed to be $x) y, g_{1}, \cdots, g_{2}, b_{2}, \cdots, b_{1}$. 
The procedure now is to "split" $x$ and obtain an imbedding of $G \vee_{K_{1}} H$ in the same surface. This yields a contradiction since $g\left(G \vee_{K_{1}} H\right)=g(G)+g(H)$.

A scheme for $G \vee_{K_{1}} H$ is constructed as follows: Replace $x$ by $x_{1}$ in all adjacency tours at vertices of $G \sim K_{2}$ and by $x_{2}$ in all adjacency tours at vertices of $H \sim K_{2}$. In the adjacency tour at $y$, replace $x$ by $x_{1}$ followed by $x_{2}$. Finally, delete the adjacency tour at $x$ and "split" it into

$$
\left.\left.x_{1}\right) y, g_{1}, \cdots, g_{2}, \quad x_{2}\right) b_{2}, \cdots, b_{1}, y .
$$

The only substantive change in the original scheme is in the adjacency tour at $y$ and at the point where the adjacency tour at $x$ was split. Hence it is easy to see that the only effect of this alteration is to split $x$ into $x_{1}$ and $x_{2}$ and enlarge the face $C$ containing the angle $g_{2} x b_{2}$ from $C=\left[g_{2}, x, b_{2}, \ldots\right]$ to $C^{\prime}=\left[g_{2}, x_{1}\right.$, $\left.y, x_{2}, b_{2}, \cdots\right]$. Hence the number of faces does not change.

Moreover, it is clear that the new scheme is for $G \vee_{K_{1}}{ }^{H}$ and so the numbers of vertices and edges each increase by one. Thus if the genus is computed using the Euler formula, it is apparent that the new scheme is for an imbedding of $G \vee_{K_{1}} H$ in a surface of the same genus as the surface given by the original scheme. But this contradicts the assumption that $G \vee_{K_{2}} H$ is imbedded in a surface of genus $g(G)+g(H)-1$.

A pictorial representation of what this splitting process looks like locally is given in Figure 6.
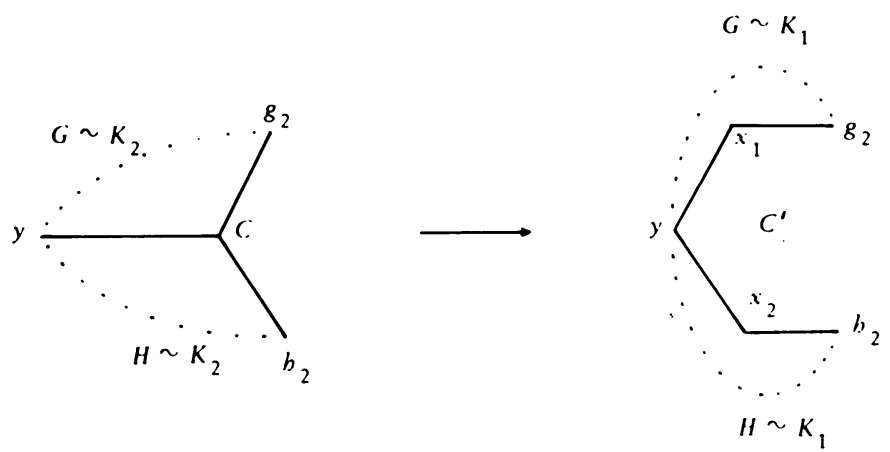

Figure 6

Finally, if $x$ is the center of precisely 2 amalgamating angles which lie on 2 distinct faces, then $y$ must also be a vertex of each of these faces. The adjacency tour at $x$ must be of the form

$$
\text { x) } y, g_{1}, \cdots, g_{2}, b_{2}, \cdots, b_{3}, g_{3}, \cdots, g_{4} \text {. }
$$

Since $y$ is also a vertex of the face with angle $g_{2} x b_{2}$ the edge $(x, y)$ can be moved from its original location to cut across that face. Once this is done, the adjacency tour at $x$ becomes 
x) $g_{1}, \cdots, g_{2}, y, b_{2}, \cdots, b_{3}, g_{3}, \cdots, g_{4}$.

Hence $G \vee_{K_{2}} H$ can be imbedded in a surface of genus $g(G)+g(H)-1$ in such a way so that $x$ is the center of precisely one amalgamating angle. The impossibility of this has just been demonstrated, and so the proof is complete.

The rest of part (1) of Theorem II.1 follows from (2) of the following theorem.

Theorem II.3. (1) Suppose that $d\left(G \vee_{K_{2}} H, g(G)+g(H)-1\right)=3$.

(a) Let $K_{2}=(x, y)$. There is an imbedding of $G \mathrm{~V}_{K_{2}}{ }^{H}$ in a surface of genus $g(G)+g(H)-1$ if and only if $G$ has a minimal imbedding for which the only nontriangular face is 7-sided of the form $\left[x, y, g_{1}, x, g_{2}, y, g_{3}\right]$ and $H$ bas a minimal imbedding for which the only nontriangular face is 7 -sided of the form $[y, x$, $\left.b_{1}, y, b_{2}, x, b_{3}\right]$.

(b) If the equivalent conditions of (a) bold, then clearly $d(G)=d(H)=4$. In particular, $G$ and $H$ are not complete (see Table I.1).

(2) If $d\left(K_{m} \vee_{K_{2}} K_{n}, g\left(K_{m}\right)+g\left(K_{n}\right)-1\right)=3$, then $g\left(K_{m} \vee K_{2} K_{n}\right)=g\left(K_{m}\right)+g\left(K_{n}\right)$.

Proof. Clearly (1a) implies (1b). Hence if (1a) is true, then $g\left(K_{m} \vee_{K_{2}} K_{n}\right) \geq$ $g\left(K_{m}\right)+g\left(K_{n}\right)$. In view of Theorem I.4, this means that (2) is a consequence of (1a).

To prove (la), assume that $G \vee_{K_{2}}{ }^{H}$ is imbedded in a surface of genus $g(G)+$ $g(H)-1$ and that $d\left(G \vee_{K_{2}} H, g(G)+g(H)-1\right)=3$. The set of nontriangular faces of the imbedding must consist of either a 6 -sided face, a pentagon and a quadrilateral, or three quadrilaterals.

By Theorem II.2(1), each vertex of $K_{2}=(x, y)$ is the center of at least 2 amalgamating angles, and if a vertex is the center of only 2 amalgamating angles, then they must lie on the same face. Because amalgamating angles cannot lie on triangular faces, and since faces with fewer than 6 sides do not have repeated vertices and a 6-sided face has at most one repeated vertex, it follows that each vertex of $K_{2}$ is the center of precisely 3 amalgamating angles, and the set of nontriangular faces must consist of 3 quadrilaterals.

Hence the adjacency tour at $x$ may be assumed to be

$$
\text { x) } y, g_{4}, \cdots, g_{1}, b_{1}, \ldots, b_{2}, g_{2}, \cdots, g_{3}, b_{3}, \ldots, b_{4} \text {. }
$$

The quadrilaterals are $Q_{1}=\left[g_{1}, x, b_{1}, y\right], Q_{2}=\left[b_{2}, x, g_{2}, y\right]$ and $Q_{3}=\left[g_{3}\right.$, $\left.x, b_{3}, y\right]$.

Because all other faces are triangular, rule $\mathrm{R}^{*}$ of Proposition $\mathrm{I} .16$ must be in force at all parts of the scheme not involving one of the $Q_{i}$ 's. The adjacency tour at $y$ must therefore take one of the following two forms:

(1) $y) g_{4}, x, b_{4}, \cdots, b_{3}, g_{3}, \cdots, g_{2}, b_{2}, \cdots, b_{1}, g_{1}, \cdots$,

(2) $y) g_{4}, x, b_{4}, \cdots, b_{1}, g_{1}, \cdots, g_{2}, b_{2}, \cdots, b_{3}, g_{3}, \cdots$

The proof proceeds by analysis in these two cases of the schemes for $G$ and 
$H$ which result when the $b_{i}$ 's or $g_{i}$ 's are deleted from the scheme for $G \vee_{K_{2}} H$. The only adjacency tours actually altered by a deletion of this type are those at $x$ and at $y$, so the computation of the new imbeddings is easy. For example, in computing the imbedding of $G$, all faces of the original imbedding not involving vertices of $H \sim K_{2}$ are carried over. In addition there will be "new" faces which correspond to the altered portions of the adjacency tours at $x$ and $y$. The only faces of the original imbedding which do not appear as a face of either the imbedding of $G$ or that of $H$ are those involving vertices from both $G \sim K_{2}$ and $H \sim K_{2}$.

These observations make it easy to compute the genus of $G \vee_{K_{2}} H$ in terms of $g(G)$ and $g(H)$. Clearly $V\left(G \vee_{K_{2}} H\right)=V(G)+V(H)-2$ and $E\left(G \vee_{K_{2}} H\right)=E(G)+$ $E(H)-1$. Let $F(G), F(H)$, and $F\left(G \vee_{K_{2}} H\right)$ denote the number of faces in the respective imbeddings of $G, H$, and $G \vee_{K_{2}} H$. Then it follows from the preceding paragraph that $F\left(G \vee_{K_{2}} H\right)$ is just $F(G)+F(H)$ less the total number $N(F)$ of "new" faces in the imbeddings of $G$ and $H$ plus the number $O(F)$ of faces in the original imbedding of $G \vee_{K_{2}}{ }^{H}$ involving vertices from both $G \sim K_{2}$ and $H \sim K_{2}$. That is, $F\left(G \vee_{K_{2}} H\right)=F(G)+F(H)-N(F)+O(F)$. If, in addition, all the imbeddings being considered are minimal, then the Euler formula gives

$$
\begin{aligned}
g(G & \left.\vee_{K_{2}} H\right)=1-1 / 2\left(V\left(G \vee_{K_{2}} H\right)-E\left(G \vee_{K_{2}} H\right)+F\left(G \vee_{K_{2}} H\right)\right) \\
= & 1-1 / 2(V(G)-E(G)+F(G))+1-1 / 2(V(H)-E(H)+F(H)) \\
& +1 / 2(N(F)-O(F)-1) \\
= & g(G)+g(H)+1 / 2(N(F)-O(F)-1) .
\end{aligned}
$$

Note that for any graph $G$ and genus $g$, if $G$ can be imbedded in a surface of genus $g$ and if $0 \leq d(G, g) \leq 5$, then the imbedding is minimal. Thus the original imbedding of $G \mathrm{~V}_{K_{2}} \mathrm{H}$ is minimal.

These ideas are now applied in the two cases mentioned above. Note that in any case $O(F)=3$ since the faces in question are $Q_{1}, Q_{2}$, and $Q_{3}$.

$$
\begin{aligned}
& \text { x) } y, g_{4}, \ldots, g_{1}, b_{1}, \ldots, b_{2}, g_{2}, \ldots, g_{3}, b_{3}, \ldots, b_{4} \text {, } \\
& \text { y) } x, b_{4}, \cdots, b_{3}, g_{3}, \cdots, g_{2}, b_{2}, \cdots, b_{1}, g_{1}, \cdots, g_{4} \text {. }
\end{aligned}
$$

Deleting the vertices of $G \sim K_{2}$ results in a scheme for $I$ with

$$
\text { x) } \left.y, b_{1}, \ldots, b_{2}, b_{3}, \ldots, b_{4}, \quad y\right) x, b_{4}, \ldots, b_{3}, b_{2}, \ldots, b_{1} \text {. }
$$

The new faces are $\left[y, x, b_{1}\right]$ and $\left[b_{2}, x, b_{3}, y\right]$. Deleting the vertices of $H \sim K_{2}$ results in a scheme for $G$ with
x) $y, g_{4}, \cdots, g_{1}, g_{2}, \cdots, g_{3}$,
y) $x, g_{3}, \cdots, g_{2}, g_{1}, \cdots, g_{4}$. 
The next faces are $\left[x, y, g_{3}\right]$ and $\left[g_{1}, x, g_{2}, y\right]$.

In each case the only nontriangular face is a quadrilateral, so the triangulation deficiency is 1 and the imbeddings are therefore minimal. Moreover, $N(F)=$ 4. Hence by $(*)$,

$$
g\left(G \vee_{K_{2}} H\right)=g(G)+g(H)+1 / 2(4-3-1)=g(G)+g(H) .
$$

Of course, this contradicts the original assumption that the imbedding of $G \vee_{K_{2}} H$ is in a surface of genus $g(G)+g(H)-1$.

$$
\begin{aligned}
& x) y, g_{4}, \ldots, g_{1}, b_{1}, \ldots, b_{2}, g_{2}, \ldots, g_{3}, b_{3}, \ldots, b_{4} \text {, } \\
& y) x, b_{4}, \ldots, b_{1}, g_{1}, \cdots, g_{2}, b_{2}, \ldots, b_{3}, g_{3}, \ldots, g_{4} \text {. }
\end{aligned}
$$

Deleting the vertices of $G \sim K_{2}$ results in a scheme for $H$ with

$$
\text { x) } \left.y, b_{1}, \ldots, b_{2}, b_{3}, \ldots, b_{4}, \quad y\right) x, b_{4}, \ldots, b_{1}, b_{2}, \ldots, b_{3} \text {. }
$$

The only new face is $\left[y, x, b_{1}, y, b_{2}, x, b_{3}\right]$.

The symmetry of the adjacency tours at $x$ and $y$ implies that when the vertices of $H \sim K_{2}$ are deleted, the only new face in the resulting imbedding of $G$ is $[x$, $\left.y, g_{1}, x, g_{2}, y, g_{3}\right]$.

These imbeddings are minimal and $N(F)=2$. Hence by $(*), g\left(G \vee_{K_{2}} H\right)=g(G)$ $+g(H)+1 / 2(2-3-1)=g(G)+g(H)-1$, as desired.

The converse proposition is clear. Given minimal imbeddings of $G$ and $H$ as in case (2), one obtains the desired scheme for $G \vee_{K_{2}} H$ by combining the schemes for $G$ and $H$ according to the recipe already set forth there. The result will be a scheme for an imbedding of $G \vee_{K_{2}} H$ in a surface of genus $g(G)+g(H)-1$. This completes the proof of Theorem II.3, and hence the proof of (1) of Theorem II.1.

Theorem II. 3 is slightly generalized by Theorem II. 4 below. For an example of a graph with a minimal imbedding of the type required by part (1a) of Theorem II.3, see Figure 7 .

C. Existence of imbeddings of $K_{m} \vee_{K_{2}} K_{n}$-Proof of (2) of Theorem II.1. According to Table II.1, the only possibility not yet considered is when $d\left(K_{m} \vee_{K_{2}} K_{n}, g\left(K_{m}\right)+g\left(K_{n}\right)-1\right)=5$.

Recall that if a complete graph has triangulation deficiency 5 , it may be a critical graph (Corollary I.13). Moreover, according to Theorem I.1, if $m=12 s+$ $5, s \geq 0$, or if $m=12 s+2$ and $s \geq 1$ is odd, then in fact $K_{m}$ is critical. This means that Theorem II.1 yields a determination of $g\left(K_{m} \vee_{K_{2}} K_{n}\right)$ in all cases except when one of $m$ and $n$ is of the form $24 s+2, s \geq 1$, and the other is congruent to 2 or 5 modulo 12 .

Part (2) of Theorem II.1 is obtained as Corollary II.5 below. 
Theorem II.4. Lèt $G$ and $H$ be amalgamated along an edge $(x, y)$. Suppose that there is a minimal imbedding of $G$ with a face $P_{1}=\left[x, y, g_{1}, \cdots, g_{n_{1}}, x, g_{n_{1}+1}\right.$, $\left.\cdots, g_{n_{2}}, y, g_{n_{2}+1}, \cdots, g_{n}\right]$ and a minimal imbedding of $H$ with a face

$$
P_{2}=\left[y, x, b_{1}, \cdots, b_{m_{1}}, y, b_{m_{1}+1}, \cdots, b_{m_{2}}, x, b_{m_{2}+1}, \cdots, b_{m}\right],
$$

where $1 \leq n_{1}<n_{2}<n$ and $1 \leq m_{1}<m_{2}<m$. Then $g\left(G \vee_{K_{2}} H\right) \leq g(G)+g(H)-1$.

Note that if $m=n=3$, this is one half of Theorem II.3(1a).

Proof. The hypothesis means that the adjacency tours at $x$ and $y$ for the imbedding of $G$ are of the form

$$
\text { x) } \left.g_{n}, y, \cdots, g_{n_{1}}, g_{n_{1}+1}, \ldots, \quad y\right) x, g_{1}, \ldots, g_{n_{2}}, g_{n_{2}+1}, \ldots
$$

Similarly, the adjacency tours at $x$ and $y$ for the imbedding of $H$ are of the form

$$
\text { x) } \left.y, b_{1}, \ldots, b_{m_{2}}, b_{m_{2}+1}, \ldots, \quad y\right) b_{m}, x, \ldots, b_{m_{1}}, b_{m_{1}+1}, \ldots
$$

A scheme for $G \vee_{K_{2}} H$ is constructed as follows. The adjacency tours at vertices of $G \sim K_{2}$ and $H \sim K_{2}$ are as they were for the original imbeddings of $G$ and $H$. The adjacency tours at $x$ and $y$ are obtained by interlacing those from the schemes for $G$ and $H$ as follows.

$$
\begin{aligned}
& \text { x) } y, \ldots, g_{n_{1}}, b_{1}, \ldots, b_{m_{2}}, g_{n_{1}+1}, \cdots, g_{n}, b_{m_{2}+1}, \ldots, \\
& \text { y) } x, \ldots, b_{m_{1}}, g_{1}, \cdots, g_{n_{2}}, b_{m_{1}+1}, \cdots, b_{m^{\prime}}, g_{n_{2}+1}, \ldots
\end{aligned}
$$

This scheme for $G \vee_{K_{2}} H$ differs from the original schemes for $G$ and $H$ only at the displayed portions of the adjacency tours at $x$ and $y$, so to compute faces one need only consider these adjacency tours. The faces are the same as for the original imbeddings of $G$ and $H$, except that $P_{1}$ and $P_{2}$ disappear and are replaced by faces

$$
\begin{aligned}
& P_{1}^{\prime}=\left[g_{n_{1}}, x, b_{1}, \ldots, b_{m_{1}}, y, g_{1}, \ldots\right], \\
& P_{2}^{\prime}=\left[b_{m_{2}}, x, g_{n_{1}+1}, \ldots, g_{n_{2}}, y, b_{m_{1}+1}, \ldots\right], \\
& P_{3}^{\prime}=\left[g_{n}, x, b_{m_{2}+1}, \ldots, b_{m}, y, g_{n_{2}+1}, \ldots\right] .
\end{aligned}
$$

The unspecified portions of these faces are the same as the corresponding portions of $P_{1}$ and $P_{2}$.

Let the numbers of faces in the imbeddings of $G, H$, and $G_{1} \vee_{K_{2}} H$ be $F(G)$, $F(H)$, and $F\left(G \vee_{K_{2}} H\right)$. Then $V\left(G \vee_{K_{2}} H\right)=V(G)+V(H)-2, E\left(G \vee_{K_{2}} H\right)=E(G)+$ $E(H)-1$, and $F\left(G \vee_{K_{2}} H\right)=F(G)+F(H)+1$. Because the imbeddings of $G$ and $H$ 
are minimal, it follows from Euler's formula that

$$
\begin{aligned}
g\left(G \vee_{K_{2}} H\right) & \leq 1-1 / 2(V(G)+V(H)-2-(E(G)+E(H)-1)+F(G)+F(H)+1) \\
& =1-1 / 2(V(G)-E(G)+F(G))-1 / 2(V(H)-E(H)+F(H)) \\
& =g(G)+g(H)-1 .
\end{aligned}
$$

Corollary II.5. If $K_{m}$ and $K_{n}$ are critical, then $g\left(K_{m} \vee_{K_{2}} K_{n}\right)=g\left(K_{m}\right)+g\left(K_{n}\right)-1$.

Proof. Recall from Corollary 1.13 that if $K_{m}$ is critical, then there is a triangular imbedding of $K_{m} \sim K_{2}$ in a surface of genus $g\left(K_{m}\right)-1$. Let $x$ and $y$ be the vertices of the deleted edge.

Since $K_{m}$ is complete, it is possible to select a vertex $z$ adjacent both to $x$ and to $y$. Hence in the triangular imbedding of $K_{m} \sim K_{2}$, there are faces $[x, z, a]$ and $[y, z, b]$ for some vertices $a$ and $b$ of $K_{m}$.

The missing edge $(x, y)$ can be restored in the following way. From the face $[x, z, a]$ delete an open 2-cell whose boundary does not intersect the boundary of the face. Remove a similarly chosen open 2-cell from the face $[y, z, b]$ and identify the resulting boundaries. This procedure adds a handle to the original surface, increasing the genus to $g\left(K_{m}\right)$. The edge $(x, y)$ can now be added, using the new handle.

When this has been done, the result is a minimal imbedding of $K_{m}$ with an 8-sided face $[x, y, z, b, y, x, z, a]$. By the same process, one obtains a minimal imbedding of $K_{n}$ with an 8-sided face $\left[x^{\prime}, z^{\prime}, y^{\prime}, b^{\prime}, z^{\prime}, x^{\prime}, y^{\prime}, a^{\prime}\right]$.

Since it does not matter which edges one uses to form $K_{m} \vee_{K_{2}} K_{n}$, we can now amalgamate by identifying the edge $(y, z)$ with the edge $\left(y^{\prime}, z^{\prime}\right)$. Then by Theorem II.4, $g\left(K_{m} \vee_{K_{2}} K_{n}\right) \leq g\left(K_{m}\right)+g\left(K_{n}\right)-1$. Since this is a lower bound for $g\left(K_{m} \vee_{K_{2}} K_{n}\right)$, this completes the proof.

Note. The imbedding of $K_{m} \vee_{K_{2}} K_{n}$ obtained in Corollary II.5 using the method of Theorem II.4 has three nontriangular faces. They are

$$
\begin{aligned}
& P_{1}=\left[b, y, b^{\prime}, z\right], \quad P_{2}=\left[x^{\prime}, y, x, z\right], \\
& P_{3}=\left[x, y, a^{\prime}, x^{\prime}, z, a\right] .
\end{aligned}
$$

This observation leads to the following result, proving (2) of Theorems I.6, I.7, and I.8.

Corollary II.6. If $K_{m}$ and $K_{n}$ are critical graphs, then for all $p$ such that $2 \leq p \leq 5, g\left(K_{m} \vee K_{p} K_{n}\right)=g\left(K_{m}\right)+g\left(K_{n}\right)-1$.

Proof. The result was established above for $p=2$. To complete the proof, note that by Kuratowski's Theorem $m, n \geq 5$, and therefore one can assume that in the above argument $a \neq b$ and $a^{\prime} \neq b^{\prime}$. This means that the other imbeddings can 
be obtained by successively collapsing the faces $P_{1}, P_{2}$, and $P_{3}$ above so as to identify $b$ with $b^{\prime}, x$ with $x^{\prime}$, and, finally, $a$ with $a^{\prime}$.

This completes the proof of Theorem II.1, but a few additional comments are in order. The results of this chapter make it tempting to conjecture that $g\left(G \vee_{K_{2}} H\right) \leq g(G)+g(H)-1$ only if $K_{2}$ or some adjacent edge is critical for $G$ and similarly for $H$.

Using Theorem II.4, it is easy to construct a counterexample to any conjecture of this type. In Figure 7 an imbedding of a 6-vertex graph $G=K_{6} \sim K_{2}$ is displayed.

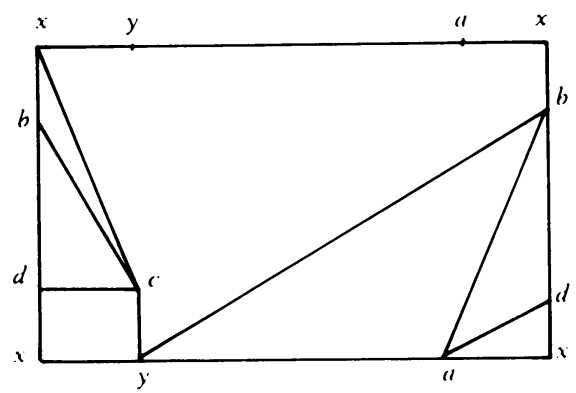

Figure 7

One face of the imbedding is the 7-sided face $[x, y, a, x, b, y, c]$. If a second copy of $G$ is imbedded in the torus as above and vertices are relabeled so that the 7-sided face becomes $\left[y^{\prime}, x^{\prime}, a^{\prime}, y^{\prime}, b^{\prime}, x^{\prime}, c^{\prime}\right]$, and if these two copies of $G$ are amalgamated along $K_{2}$ by identifying $x$ with $x^{\prime}$ and $y$ with $y^{\prime}$, then it follows from Theorem II.4 that in this case $g\left(G \vee_{K_{2}} G\right)=1$. Now $V(G)=6$ and $E(G)=14$ so removing any edge from $G$ and applying Corollary I.10,

$$
\begin{aligned}
g\left(G \sim K_{2}\right) & \geq\left\{E\left(G \sim K_{2}\right) / 6-V\left(G \sim K_{2}\right) / 2+1\right\} \\
& =\{13 / 6-6 / 2+1\}=\{(13-18+6) / 6\}=1 .
\end{aligned}
$$

Thus no edge of $G$ is critical.

The only case in which $g\left(K_{m} \vee_{K_{2}} K_{n}\right)$ is not determined by Theorem II.1 is when one of $m$ and $n$ is of the form $24 s+2, s \geq 1$, and the other is congruent to 2 or 5 modulo 12. Finding the genus in this case might involve more than determining whether or not $K_{24 s+2}$ is critical, as the preceding example shows. Indeed, it may happen that $K_{24 s+2}$ is not critical but nevertheless has a minimal imbedding with a 7 -sided face of the desired type.

As a final remark, notice that determining whether or not a complete graph is critical is actually a problem in amalgamations of complete graphs, since $K_{m} \sim K_{2}$ $=K_{m-1} \vee_{K_{m-2}} K_{m-1}$. 


\section{AMALGAMATIONS ALONG $K_{p}, p \geq 3$}

A. Restatement of theorems and analysis of cases $p=3$ and 4 . The first sections of this chapter are devoted to the proof of Theorems 1.6 and I.7, which will now be restated as Theorems III.1 and III:2.

Theorem III.1. (1) If $g\left(K_{m} \vee K_{3} K_{n}\right)=g\left(K_{m}\right)+g\left(K_{n}\right)-1$, then one of $m$ and $n$ is congruent to 2 or 5 modulo 12, and the other is congruent to 1, 2, 5, 6, 9 or 10 modulo 12.

(2) If $K_{m}$ and $K_{n}$ are critical, then $g\left(K_{m} \vee_{K_{3}} K_{n}\right)=g\left(K_{m}\right)+g\left(K_{n}\right)-1$.

(3) If $K_{m}$ is critical and $K_{n}$ is congruent to $1,6,9$ or 10 modulo 12, then $g\left(K_{m} \vee K_{3} K_{n}\right)=g\left(K_{m}\right)+g\left(K_{n}\right)-1$ if and only if $K_{n}$ bas a minimal imbedding with a 6-sided face which has a repeated vertex. By symmetry, the same statement with the roles of $m$ and $n$ interchanged is also true.

Theorem III.2. (1) If $g\left(K_{m} \vee K_{4} K_{n}\right)=g\left(K_{m}\right)+g\left(K_{n}\right)-1$, then one of $m$ and $n$ is congruent to 2 or 5 modulo 12 and the other is congruent to $1,2,5,6,8,9,10$, or 11 modulo 12.

(2) If $K_{m}$ and $K_{n}$ are critical, then $g\left(K_{m} \vee_{K_{4}} K_{n}\right)=g\left(K_{m}\right)+g\left(K_{n}\right)-1$.

(3) If one of $m$ and $n$ is congruent to 5 modulo 12 and the other is congruent to $1,2,5,6,8,9,10$, or 11 modulo 12 , then $g\left(K_{m} \vee K_{4} K_{n}\right)=g\left(K_{m}\right)+g\left(K_{n}\right)-1$.

In combination with Theorems $\mathrm{I} .1$ and I.4, these theorems determine the genera of graphs of the form $K_{m} \vee_{K_{p}} K_{n}$ with $p=3$ or 4 in most cases. The genera are unknown in the remaining cases.

Table III.1

$$
d\left(K_{m} \vee_{K_{p}} K_{n^{\prime}} g\left(K_{m}\right)+g\left(K_{n}\right)-1\right)
$$

as a function of $d\left(K_{m}\right)$ and $d\left(K_{n}\right), p=3$ and 4

\begin{tabular}{r|r|r|r|r|}
$d\left(K_{m}\right)$ & 0 & 2 & 3 & 5 \\
\hline 0 & -6 & -4 & -3 & -1 \\
2 & -4 & -2 & -1 & 1 \\
3 & -3 & -1 & 0 & 2 \\
5 & -1 & 1 & 2 & 4
\end{tabular}


As in Chapter II, the proofs are by cases, depending on the value of the triangulation deficiency of $K_{m} \vee K_{p} K_{n}$ for genus $g\left(K_{n}\right)+g\left(K_{n}\right)-1$. Since $d\left(K_{3}\right)=$ $d\left(K_{4}\right)=0$, the values of $d\left(K_{m} \vee K_{p} K_{n}, g\left(K_{m}\right)+g\left(K_{n}\right)-1\right)$ are the same for $p=3$ and $p=4$, and are displayed in Table III.1. The values are computed by applying Proposition I.11. By Proposition I.9, if the value of the triangulation deficiency is negative, then an imbedding is impossible. Thus there are only four cases to consider, namely when $d\left(K_{m} \bigvee_{K_{p}} K_{n}, g\left(K_{m}\right)+g\left(K_{n}\right)-1\right)$ is $0,1,2$ or 4.

Parts $(\angle)$ of Theorem III. 1 and III. 2 were proven as Corollary II.6. $\$$ III.B is devoted to proving the impossibility of imbedding $K_{m} \vee_{K_{p}} K_{n}$ in a surface of genus $g\left(K_{m}\right)+g\left(K_{n}\right)-1$ when $p=3$ and the triangulation deficiency is 0 or 1 and when $p=4$ and the triangulation deficiency is 0 . This will prove (1) of Theorems III.1 and III.2. Finally, part (3) of Theorem III.1 is proven in $\$$ III.C and part (3) of Theorem III. 2 is proven in $\$$ III.D. As in Chapter II, results are stated for arbitrary amalgamations of graphs whenever possible.

B. Nonexistence of imbeddings of $K_{m}{ }^{V} K_{p} K_{n}, p=3$ or 4 . The notational conventions of Chapter II will also be used in this chapter (see $\$$ II.B).

Part (1) of Theorem III.1 is a consequence of (2) of the following theorem.

Theorem III.3. (1) Suppose that $G \vee_{K_{3}} \mathrm{H}$ bas a 2-cell imbedding in a surface of genus $g$ such that a vertex $x \in K_{3}$ is not the center of any amalgamating angle. Then $G \vee_{K_{2}} H$ can also be imbedded in a surface of genus $g$ and $d\left(G \vee_{K_{2}} H, g\right)=$ $d\left(G \vee_{K_{3}} H, g\right)+1$.

(2) If $G \vee_{K_{3}} H$ can be imbedded in a surface of genus $g(G)+g(H)-1$, then $d\left(G \vee_{K_{3}} H, g(G)+g(H)-1\right) \geq 2$.

Proof. Of course, the main interest here is in (2), so first it will be shown that (2) is a consequence of (1).

To see this, assume that (1) is true and that $G \vee_{K_{3}} H$ is imbedded in a surface of genus $g(G)+g(H)-1$. If $d\left(G \vee_{K_{3}} H, g(G)+g(H)-1\right) \leq 1$, then the imbedding is a 2 -cell imbedding and there is at most one nontriangular face. If there is a nontriangular face, it must be a quadrilateral. Because amalgamating angles can occur only on nontriangular faces, this means that some vertex of $K_{3}$ is not the center of any amalgamating angle. Now (1) implies that $G \vee_{K_{2}}{ }^{H}$ can also be imbedded in a surface of genus $g(G)+g(H)-1$ and furthermore that $d\left(G \vee_{K_{2}} H, g(G)+g(H)-1\right) \leq 2$. This contradicts Theorem II.2 (2) and so $d\left(G \vee_{K_{3}} H, g(G)+g(H)-1\right) \geq 2$. 
To prove (1), let $G \vee_{K_{3}} H$ have a 2-cell imbedding in a surface of genus $g$ such that a vertex $x \in K_{3}$ is not the center of any amalgamating angle. The idea of the proof is to "split" $x$ into 2 new vertices $x_{1}$ and $x_{2}$ in such a way so as to give the desired imbedding of $G \vee_{K_{2}} H$.

Suppose first that $x$ is not adjacent to any vertex of $G \sim K_{3}$. Then $G \vee_{K_{3}} H=$ ( $G \sim x) \vee_{K_{2}} H$ where $G \sim x$ is defined to be the subgraph of $G$ obtained by removing the vertex $x$ and all edges on which it is incident. If $y$ and $z$ are the other vertices of $K_{3}$, then a vertex $x^{\prime}$ can be added to the interior of any face with $(y, z)$ as a side, along with edges $\left(x^{\prime}, y\right)$ and $\left(x^{\prime}, z\right)$. This gives the desired imbedding of $G \vee_{K_{2}} H$.

The same method yields the desired imbedding of $\mathrm{GV}_{\mathrm{K}_{2}}{ }^{H}$ if $x$ is not adjacent to any vertex of $H \sim K_{3}$. So assume that $x$ is adjacent to at least one vertex of $G \sim K_{3}$ and one of $H \sim K_{3}$.

Because $x$ is not the center of any amalgamating angle, the adjacency tour at $x$ is of the form

$$
\text { x) } y, g_{1}, \cdots, g_{2}, z, b_{1}, \ldots, b_{2} \text {. }
$$

An imbedding of $G \vee_{K_{2}} H$ in a surface of genus $g$ is obtained by splitting $x$ into two new vertices $x_{1}$ and $x_{2}$ as follows. In all adjacency tours at vertices of $G \sim K_{3}$ change $x$ to $x_{1}$; in all adjacency tours at vertices of $H \sim K_{3}$ change $x$ to $x_{2}$. Replace $x$ by $x_{1}, x_{2}$ in the adjacency tour at $y$ and by $x_{2}, x_{1}$ in the adjacency tour at $z$. Finally, "split" the adjacency tour at $x$ into the adjacency tours

$$
\left.\left.x_{1}\right) y, g_{1}, \cdots, g_{2}, z, \quad x_{2}\right) z, b_{1}, \cdots, b_{2}, y .
$$

Clearly this gives a scheme for $\mathrm{GV}_{\mathrm{K}_{2}} \mathrm{H}$. The only substantive differences between this and the original scheme are in the adjacency tours at $y$ and $z$ and at the point where the adjacency tour at $x$ has been split into those at $x_{1}$ and $x_{2}$. Hence all the faces of the original imbedding are faces of the new one, and in addition there is a new quadrilateral $\left[z, x_{1}, y, x_{2}\right]$. This means that the number of faces and the triangulation deficiency have each increased by 1 (this is actually a consequence of the definition of triangulation deficiency).

The change from $G V_{K_{3}} H$ to $G V_{K_{2}} H$ has increased the number of vertices by 1 and the number of edges by 2 . It therefore follows from the Euler formula that the genus remains unchanged. This completes the proof of the theorem and hence of part (1) of Theorem III.1.

In order to give the reader an intuitive feeling for the splitting process used above, it is illustrated in Figure 8. 

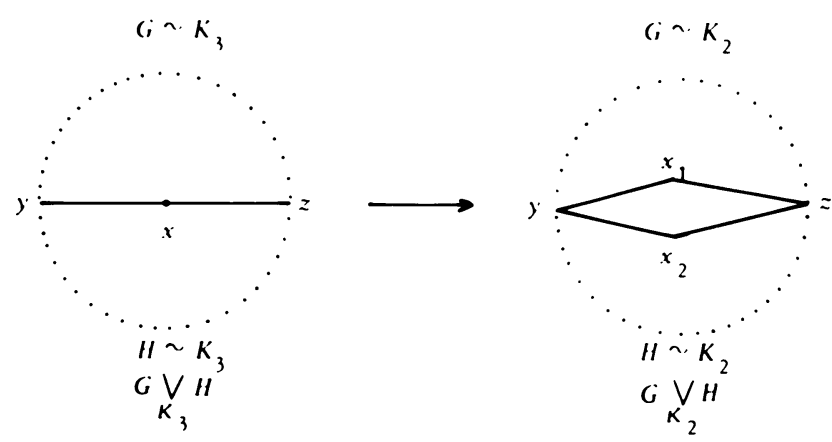

Figure 8

Part (1) of Theorem III. 2 follows from the second assertion of the next theorem.

Theorem III.4. Let $K_{4}$ bave vertices $x, y, z, w$. A graph $G \bigvee_{K_{4}}{ }^{H}$ can be triangularly imbedded in a surface of genus $g(G)+g(H)-1$, if and only if one of $G, H$ has a minimal imbedding for which the only nontriangular face is 8-sided of the form $[w, x, y, z, x, w, z, y]$ and the other has a minimal imbedding for which the only nontriangular face is a quadrilateral $[y, x, z, w]$. In particular, if $d\left(K_{m} \vee_{K_{4}} K_{n}, g\left(K_{m}\right)+g\left(K_{n}\right)-1\right)=0$, then $g\left(K_{m} \vee_{K_{4}} K_{n}\right)=g\left(K_{m}\right)+g\left(K_{n}\right)$.

Proof. Recall that $g\left(K_{m} \vee K_{4} K_{n}\right)$ is either $g\left(K_{m}\right)+g\left(K_{n}\right)$ or $g\left(K_{m}\right)+g\left(K_{n}\right)-1$ and that a complete graph can never have triangulation deficiency 1 . Hence the second assertion follows from the first.

The rest of the proof follows the same pattern as in the proof of Theorem II.3.

Suppose that a scheme for a triangular imbedding of $G \mathrm{~V}_{K_{4}} \mathrm{H}$ in a surface of genus $g(G)+g(H)-1$ is given. The vertices of $K_{4}$ will be denoted $x, y, z, w$. Recall that there can be no amalgamating angles in a triangular imbedding.

Each vertex of $K_{4}$ must be adjacent to at least one vertex of $G \sim K_{4}$ and one vertex of $H \sim K_{4}$. Otherwise, say $x$ is not adjacent to any vertex of $G \sim K_{4}$. Then $G \vee_{K_{4}} H=(G \sim x) \vee_{K_{3}} H$, and by Theorem III.3 (1) an imbedding of $(G \sim x) \vee_{K_{2}} H$ in a surface of genus $g(G)+g(H)-1$ can be obtained, say by splitting the vertex $y$ into vertices $y_{1} \in G$ and $y_{2} \in H$. Some face $P$ of this imbedding will have $w, z$, and $y_{1}$ as vertices. If a new vertex $x_{1}$ and edges between $x_{1}$ and $z, w$, and $y_{1}$ are placed inside $P$, the result is an imbedding of $G \vee_{K_{2}} H$ in a sur face of genus $g(G)+g(H)-1$. Since $V\left(G \vee_{K_{2}} H\right)=V\left(G \vee_{K_{4}} H\right)+2$ and $E\left(G \vee_{K_{2}} H\right)=E\left(G \vee_{K_{4}} H\right)+5$, if $d\left(G \vee_{K_{4}} H, g(G)+g(H)-1\right)=0$, then $d\left(G \vee_{K_{2}} H, g(G)+g(H)-1\right)=1$, contradicting Theorem II.2 (2). Thus each vertex of $K_{4}$ is adjacent to at least one vertex of $G \sim K_{4}$ and one vertex of $H \sim K_{4}$.

Furthermore, no triangular subgraph of $K_{4}$ may bound a face of the imbedding. Indeed, if the $K_{3}$ with vertices $x, y$ and $z$ bounds a face, then the adjacency tour at $x$ must be of the form 


$$
\text { x) } y, z, g_{1}, \ldots, g_{2}, w, b_{1}, \ldots, b_{2} \text {. }
$$

Because rule $\mathrm{R}^{*}$ of Proposition $\mathrm{I} .16$ holds, the adjacency tour at $y$ must then be of the form

$$
y) z, x, b_{2}, \ldots, b_{3}, w, g_{3}, \cdots, g_{4}
$$

and that at $z$ must be in part

$$
\text { z) } g_{1}, x, y, g_{4}, \ldots \text {. }
$$

Since $z$ cannot be the center of any amalgamating angle, there is no way to complete the adjacency tour at $z$ so as to make $z$ adjacent to a vertex of $H \sim K_{4}$, a contradiction.

These considerations show that one can assume without loss of generality that the adjacency tour at $x$ is

$$
\text { x) } y, g_{1}, \ldots, g_{2}, z, b_{1}, \ldots, b_{2}, w, b_{3}, \ldots, b_{4} \text {. }
$$

Now the fact that $w$ is not the center of an amalgamating angle and rule $\mathrm{R}^{*}$ imply that the adjacency tour at $w$ is in one of the following two forms.

$$
\text { w) } b_{3}, x, b_{2}, \ldots, b_{5}, y, g_{3}, \ldots, g_{4}, z, b_{6},
$$$$
\text { w) } b_{3}, x, b_{2}, \ldots, b_{5}, z, g_{3}, \cdots, g_{4}, y, b_{6} \text {. }
$$

If the adjacency tour at $w$ is as in (1), then $b_{1}, x$, and $g_{2}$ are successive in the adjacency tour at $z$, as are $b_{6}, w$, and $g_{4}$ by $\mathrm{R}^{*}$. But if this is true, then $z$ is the center of an amalgamating angle, a contradiction. So the adjacency tour at $w$ must be as in (2).

The same reasoning applied to the adjacency tours at $y$ and $z$ shows that there are two possible configurations for the adjacency tours at $x, y, z$ and $w$ :

$$
\begin{aligned}
& \text { x) } y, g_{1}, \ldots, g_{2}, z, b_{1}, \ldots, b_{2}, w, b_{3}, \ldots, b_{4} \text {, } \\
& \text { y) } g_{1}, x, b_{4}, \ldots, b_{6}, w, g_{4}, \ldots, g_{5}, z, g_{6}, \ldots, \\
& z) b_{1}, x, g_{2}, \ldots, g_{6}, y, g_{5}, \cdots, g_{3}, w, b_{5}, \ldots, \\
& \text { w) } b_{3}, x, b_{2}, \ldots, b_{5}, z, g_{3}, \cdots, g_{4}, y, b_{6}, \ldots ;
\end{aligned}
$$

$$
\begin{aligned}
& \text { x) } y, g_{1}, \ldots, g_{2}, z, b_{1}, \ldots, b_{2}, w, b_{3}, \ldots, b_{4} \text {, } \\
& \text { y) } g_{1}, x, b_{4}, \ldots, b_{7}, z, b_{8}, \ldots, b_{6}, w, g_{4}, \ldots, \\
& z) b_{1}, x, g_{2}, \cdots, g_{3}, w, b_{5}, \ldots, b_{8}, y, b_{7}, \ldots, \\
& \text { w) } b_{3}, x, b_{2}, \ldots, b_{5}, z, g_{3}, \cdots, g_{4}, y, b_{6}, \ldots
\end{aligned}
$$


The procedure now is to analyze the imbeddings of $G$ and $H$ which result when the vertices of $H \sim K_{4}$ and $G \sim K_{4}$ are deleted in each of the 2 cases. Such a deletion only alters the adjacency tours at $x, y, z$ and $w$ and so all faces come from the original imbedding, except where the adjacency tours at $K_{4}$ have been changed. To compute the new faces, one need only examine these adjacency tours (for more details see the proof of Theorem II.3).

(1) When the vertices of $H \sim K_{4}$ are deleted, the resulting scheme for $G$ has adjacency tours
x) $y, g_{1}, \cdots, g_{2}, z, u$,
y) $g_{1}, x, w, g_{4}, \cdots, g_{5}, z, g_{6}$,
z) $x, g_{2}, \cdots, g_{6}, y, g_{5}, \cdots, g_{3}, w$,
w) $x, z, g_{3}, \cdots, g_{4}, y$.

The new faces are $[z, x, w]$ and $[x, y, w]$.

Deleting the vertices of $G \sim K_{4}$, one obtains a scheme for $H$ with

x) $\left.y, z, b_{1}, \ldots, b_{2}, w, b_{3}, \cdots, b_{4}, \quad y\right) x, b_{4}, \ldots, b_{6}, w, z$,

z) $b_{1}, x, y, w, b_{5}, \ldots, \quad$ w) $b_{3}, x, b_{2}, \ldots, b_{5}, z, y, b_{6}, \ldots$

The new faces are $[y, x, z]$ and $[z, w, y]$.

Both of the se imbeddings are triangular and therefore minimal. Clearly, when they are put together to form the original scheme for $G \vee_{K_{4}} H$, no new faces are created and the 4 "new" triangles $[z, x, w],[x, y, w],[y, x, z]$, and $[z, w, y]$ are lost. In fact, this process is the same as that used in the proof of Proposition I.15 in the special case $p=4$.

If $F(G), F(H)$, and $F\left(G \vee K_{4} H\right)$ denote the number of faces in the corresponding imbeddings, it has just been observed that $F\left(G \vee_{K_{4}} H\right)=F(G)+F(H)-4$. Moreover, by construction $V\left(G \vee_{K_{4}}{ }^{H}\right)=V(H)+V(H)-4$ and $E\left(G \vee_{K_{4}} H\right)=E(G)+$ $E(H)-6$. Hence by the modified Euler formula

$$
\begin{aligned}
g\left(G \vee_{K_{4}} H\right) & =1-1 / 2(V(G)+V(H)-4-(E(G)+E(H)-6)+F(G)+F(H)-4) \\
& =1-1 / 2(V(G)-E(G)+F(G))+1-1 / 2(V(H)-E(H)+F(H)) \\
& =g(G)+g(H) .
\end{aligned}
$$

Since this contradicts the original assumption, case $(1)$ is impossible.

(2) If the vertices of $H \sim K_{4}$ are deleted, the resulting scheme for $G$ has

$$
\begin{aligned}
& \text { x) } \left.y, g_{1}, \cdots, g_{2}, z, w, \quad y\right) g_{1}, x, z, w, g_{4}, \ldots, \\
& \left.z) x, g_{2}, \cdots, g_{3}, w, y, \quad w\right) x, z, g_{3}, \cdots, g_{4}, y .
\end{aligned}
$$

The only new face is $[w, x, y, z, x, w, z, y]$.

Deleting the vertices of $G \sim K_{4}$ results in a scheme for $H$ with 


$$
\begin{aligned}
& \text { x) } y, z, b_{1}, \ldots, b_{2}, w, b_{3}, \ldots, b_{4} \text {, } \\
& \text { y) } x, b_{4}, \ldots, b_{7}, z, b_{8}, \ldots, b_{6}, w, \\
& z) b_{1}, x, w, b_{5}, \ldots, b_{8}, y, b_{7}, \ldots, \\
& w) b_{3}, x, b_{2}, \ldots, b_{5}, z, y, b_{6}, \ldots .
\end{aligned}
$$

The only new face is $[y, x, z, w]$.

It is clear that when these schemes are put together to form the original scheme for $G \vee_{K_{4}} H$ no new faces are created, and the faces $[w, x, y, z, x, w, z, y]$ and $[y, x, z, w]$ are lost. Moreover, since the triangulation deficiencies are at most 5, the imbeddings are minimal. A computation using the modified Euler formula as in case (1) therefore shows that $g\left(G \vee_{K_{4}} H\right)=g(G)+g(H)-1$, as desired.

Finally, note that if minimal imbeddings of $G$ and $H$ with nontriangular faces $[w, x, y, z ; x, w, z, y]$ and $[y, x, z, w]$, respectively, are given, then the adjacency tours at $x, y, z$ and $w$ must be as in (2) above. Combining the se adjacency tours according to the recipe given by (2) yields a scheme for the desired imbedding of $G \vee_{K_{4}} H$ in a surface of genus $g(G)+g(H)-1$. A more topological statement of this procedure is easy to give and provides the basis for the proof of Theorem III.8.

C. An ambiguous case-Proof of (3) of Theorem III.1. Part (3) of Theorem III.1 follows from the next theorem, whose proof is similar to that of Theorem III.4.

Theorem III.5. Suppose that $d\left(G \vee_{K_{3}} H, g(G)+g(H)-1\right)=2$. Then $g\left(G \vee_{K_{3}} H\right)=g(G)+g(H)-1$ if and only if $G$ bas a minimal imbedding for which the only nontriangular face is 8-sided of the form $\left[g_{1}, x, y, z, g_{2}, x, z, y\right]$ and $H$ bas a minimal imbedding for which the only nontriangular face is 6-sided of the form $\left[y, x, b_{2}, z, x, b_{1}\right]$, where $x, y$, and $z$ are the vertices of $K_{3}$.

In particular, if $d\left(K_{m} \vee K_{3} K_{n}, g\left(K_{m}\right)+g\left(K_{n}\right)-1\right)=2$, then $g\left(K_{m} \vee K_{3} K_{n}\right)=$ $g\left(K_{m}\right)+g\left(K_{n}\right)-1$ if and only if one of $K_{m}$ and $K_{n}$ is critical and the other has a minimal imbedding for which the only nontriangular face is 6-sided with a repeated vertex.

Proof. The last statement follows from the first, since an 8-sided face with a repeated edge $e$ can be cut open along that edge to give an imbedding of $G \sim e$ in a surface of genus one less than the genus of the original surfaces.

To prove the first assertion, suppose that $G \vee_{K_{3}} H$ is imbedded in a surface of genus $g(G)+g(H)-1$ and that $d\left(G \vee_{K_{3}} H, g(G)+g(H)-1\right)=2$. By Theorems III.3(1) and II.2(2) each vertex of $K_{3}$ is the center of at least one amalgamating. angle. Because an amalgamating angle cannot lie on a triangular face, this means that the set of nontriangular faces of the imbedding must consist of quadrilaterals 
$Q_{1}$ and $Q_{2}$, each having diagonally opposite amalgamating angles. One of the vertices of $K_{3}$, say $x$, must therefore be the center of 2 amalgamating angles, one on $Q_{1}$ and the other on $Q_{2}$.

Note that if $K_{3}$ actually bounds a face of the imbedding, then the edges $(x, y)$ and $(x, z)$ may be moved to the quadrilaterals $Q_{1}$ and $Q_{2}$. This gives an imbedding of $G_{K_{3}} H$ in the same surface such that neither $y$ nor $z$ is the center of any amalgamating angle, a contradiction.

Combining these facts, one sees that the adjacency tour at $x$ must take one of the following four forms:

$$
\begin{aligned}
& \text { x) } g_{1}, b_{1}, \ldots, b_{3}, y, g_{3}, \ldots, g_{2}, b_{2}, \ldots, b_{4}, z, g_{4}, \ldots, \\
& \text { x) } g_{1}, b_{1}, \ldots, b_{2}, g_{2}, \ldots, g_{3}, y, b_{3}, \ldots, b_{4}, z, g_{4}, \ldots, \\
& \text { x) } g_{1}, b_{1}, \ldots, b_{3}, y, b_{4}, \ldots, b_{2}, g_{2}, \ldots, g_{3}, z, g_{4}, \ldots, \\
& \text { x) } g_{1}, b_{1}, \ldots, b_{2}, g_{2}, \ldots, g_{3}, y, g_{4}, \ldots, g_{5}, z, g_{6}, \ldots
\end{aligned}
$$

Note that in cases (3) and (4), the edges $(x, y)$ and $(x, z)$ can be moved to the quadrilaterals $Q_{1}$ and $Q_{2}$ to yield an imbedding of $G V_{K_{3}} H$ in the same surface such that $x$ is not the center of any amalgamating angles, which is a contradiction. Hence these cases cannot occur.

The procedure now is to analyze the schemes for $G$ and $H$ which result in cases (1) and (2) when the vertices of $H \sim K_{3}$ and $G \sim K_{3}$ are deleted. The only altered adjacency tours will be those at $x, y$, and $z$, and hence only these parts of the new schemes need be considered. Rule $\mathrm{R}^{*}$ applies to all parts of the schemes for $G \vee_{K_{3}} H$ not giving angles of $Q_{1}$ or $Q_{2}$.

(1) If $Q_{1}=\left[y, g_{1}, x, b_{1}\right]$ and $Q_{2}=\left[z, g_{2}, x, b_{2}\right]$, then thè adjacency tours at $x, y$, and $z$ must be in one of the two forms

$$
\text { x) } g_{1}, b_{1}, \ldots, b_{3}, y, g_{3}, \cdots, g_{2}, b_{2}, \ldots, b_{4}, z, g_{4}, \ldots \text {, }
$$

y) $b_{1}, g_{1}, \ldots, g_{3}, x, b_{3}, \cdots, b_{5}, z, b_{6}, \cdots$,

z) $b_{2}, g_{2}, \cdots, g_{4}, x, b_{4}, \cdots, b_{6}, y, b_{5}, \cdots$;

x) $g_{1}, b_{1}, \ldots, b_{3}, y, g_{3}, \ldots, g_{2}, b_{2}, \ldots, b_{4}, z, g_{4}, \ldots$,

$$
\begin{aligned}
& \text { y) } b_{1}, g_{1}, \ldots, g_{5}, z, g_{6}, \ldots, g_{3}, x, b_{3}, \ldots, \\
& z) b_{2}, g_{2}, \cdots, g_{6}, y, g_{5}, \ldots, g_{4}, x, b_{4}, \ldots
\end{aligned}
$$

In addition, there are two subcases where $Q_{1}=\left[z, g_{1}, x, b_{1}\right]$ and $Q_{2}=$ $\left[y, g_{2}, x, b_{2}\right]$. However, it is clear from the symmetry of the adjacency tour at $x$ that these will be the same as in (a) and (b) with the roles of $y$ and $z$ interchanged, and so need not be considered separately. 
(a) Deleting the vertices of $H \sim K_{3}$, one obtains a scheme for $G$ with

$$
\begin{aligned}
& \text { x) } g_{1}, y, g_{3}, \ldots, g_{2}, z, g_{4}, \cdots, \\
& \text { y) } g_{1}, \ldots, g_{3}, x, z, \\
& \text { z) } g_{2}, \cdots, g_{4}, x, y \cdot
\end{aligned}
$$

The only new face is $\left[g_{1}, x, y, z, g_{2}, x, z, y\right]$.

Deleting the vertices of $G \sim K_{3}$, one obtains a scheme for $H$ with

$$
\begin{aligned}
& \text { x) } b_{1}, \cdots, b_{3}, y, b_{2}, \cdots, b_{4}, z \text {, } \\
& \text { y) } b_{1}, x, b_{3}, \cdots, b_{5}, z, b_{6}, \cdots, \\
& z) b_{2}, x, b_{4}, \cdots, b_{6}, y, b_{5}, \cdots
\end{aligned}
$$

The only new face is $\left[y, x, b_{2}, z, x, b_{1}\right]$.

In both cases the triangulation deficiency is at most 5 and so the imbeddings are minimal. Moreover, when these schemes are combined so as to form the original scheme for $G \vee_{K_{3}} H$, the faces $\left[g_{1}, x, y, z, g_{2}, x, z, y\right]$ and $\left[y, x, b_{2}, z, x, b_{1}\right]$ disappear and $Q_{1}$ and $Q_{2}$ are created. Thus there is no net change in the total number of faces. Three vertices and three edges are lost when $G$ and $H$ are amalgamated along $K_{3}$. In this case, then, the Euler formula implies that $g\left(G \vee_{K_{3}} H\right)=$ $g(G)+g(H)-1$.

Because the above construction is completely reversible, this case gives the conclusion of the theorem. It remains either to eliminate the other cases or to show that they yield the same result.

(b) Deleting the vertices of $H \sim K_{3}$, one obtains a scheme for $G$ with

$$
\begin{aligned}
& \text { x) } g_{1}, y, g_{3}, \ldots, g_{2}, z, g_{4}, \ldots, \\
& \text { y) } g_{1}, \ldots, g_{5}, z, g_{6}, \ldots, g_{3}, x, \\
& \text { z) } g_{2}, \cdots, g_{6}, y, g_{5}, \ldots, g_{4}, x .
\end{aligned}
$$

The new faces are $\left[g_{1}, x, y\right]$ and $\left[g_{2}, x, z\right]$.

Deleting the vertices of $G \sim K_{3}$ yields a scheme for $H$ with

$$
\begin{aligned}
& \text { x) } b_{1}, \ldots, b_{3}, y, b_{2}, \cdots, b_{4}, z, \\
& \text { y) } b_{1}, z, x, b_{3}, \cdots, \\
& z) b_{2}, y, x, b_{4}, \cdots
\end{aligned}
$$

The new faces are $\left[z, x, b_{1}, y\right]$ and $\left[y, x, b_{2}, z\right]$.

These schemes are for minimal imbeddings of $G$ and $H$. When they are combined to form the original imbedding of $G \vee_{K_{3}} H$, the faces $\left[g_{1}, x, y\right],\left[g_{2}, x, z\right]$, 
$\left[z, x, b_{1}, y\right]$ and $\left[y, x, b_{2}, z\right]$ are lost and $Q_{1}$ and $Q_{2}$ are created. Hence the total number of faces decreases by 2 . In this case, then, the Euler formula implies that the imbedding of $G \vee_{K_{3}} H$ is in a surface of genus $g(G)+g(H)$, a contradiction. This configuration is, therefore, impossible.

(2) In this case, the adjacency tour at $x$ is $x) g_{1}, b_{1}, \cdots, b_{2}, g_{2}, \cdots, g_{3}, y$, $b_{3}, \cdots, b_{4}, z, g_{4}, \cdots$.

Rule $\mathrm{R}^{*}$ applies to all parts of the scheme not giving angles of $Q_{1}$ or $Q_{2}$, so the adjacency tour at $y$ must be in part $y) b_{3}, x, g_{3}, \cdots$.

Now $y$ is the center of exactly one amalgamating angle, either $g_{2}, y, b_{2}$ or $b_{1}, y, g_{1}$. In the latter case, the form of the adjacency tour at $y$ would force $y$ to be the center of at least one more amalgamating angle, a contradiction. Hence $Q_{1}=\left[z, g_{1}, x, b_{1}\right]$ and $Q_{2}=\left[y, g_{2}, x, b_{2}\right]$.

If the edge $(x, y)$ is moved from its present location to become a diagonal of $Q_{2}$, then the adjacency tour at $x$ becomes: $\left.x\right) g_{1}, b_{1}, \cdots, b_{2}, y, g_{2}, \cdots, g_{3}, b_{3}, \cdots$, $b_{4}, z, g_{4}, \cdots$.

This is the same as in case (1). Thus the adjacency tour at $x$ is as in (2) if and only if there is an imbedding of $G_{K_{3}} H$ in a surface of the same genus with the adjacency tour at $x$ as in (1). This case has already been analyzed, and so the proof of the theorem is complete, as is the proof of Theorem III.1.

This case actually remains ambiguous because it is only known in certain special cases whether $d\left(K_{n}\right)=3$ implies $K_{n}$ has a minimal imbedding such that there is a 6-sided face with a repeated vertex. Furthermore, there is still the difficulty of determining whether or not $K_{24 s+2}$ is critical, $s \geq 1$. Some examples follow.

Proposition III.6. There is no minimal imbedding of $K_{6}$ for which one face is 6-sided with a repeated vertex.

Proof. Suppose that such an imbedding of $K_{6}$ exists and that the 6-sided face is $H=[a, b, x, c, d, x]$. If the other vertex of $K_{6}$ is $e$, then the adjacency tour at $x$ must be in one of the two forms

$$
\begin{aligned}
& x) d, a, b, c, e, \\
& x) d, a, e, b, c .
\end{aligned}
$$

Since $d\left(K_{6}\right)=3$, all other faces are triangular and so rule R2 of Proposition I.16 holds at all parts of the scheme not related to $H$.

In case ( 1 ), this means that the adjacency tour at a must be a) $b, x, \cdots$, contradicting the fact that $x a b$ is an angle of $H$.

In case (2), the adjacency tour at $c$ must be $c) d, x, \cdots$, contradicting the fact that $x<d$ is an angle of $H$. 
Corollary III.7. If $m$ is congruent to 2 or 5 modulo 12 , then $g\left(K_{m} \vee_{K_{3}} K_{6}\right)=$ $g\left(K_{m}\right)+1$.

On the other hand, Mayer [7] has found minimal imbeddings of $K_{9}$ and $K_{18}$ having 6-sided faces with repeated vertices. Thus if $K_{m}$ is critical, $g\left(K_{m} \vee_{K_{3}} K_{9}\right)=g\left(K_{m}\right)+g\left(K_{9}\right)-1=g\left(K_{m}\right)+2$ and $g\left(K_{m} \vee_{K_{3}} K_{18}\right)=g\left(K_{m}\right)+$ $g\left(K_{18}\right)-1=g\left(K_{m}\right)+17$.

D. Special constructions. If a complete graph $K_{m}$ is critical, then $K_{m} \sim K_{2}$ can be triangularly imbedded in a surface of genus $g\left(K_{m}\right)-1$. This observation can be combined with the construction used to prove Proposition I.15 to give some special constructions of imbeddings of $K_{m} \vee K_{p} K_{n}$ when one or both of $K_{m}$ and $K_{n}$ is critical. In particular, constructions of this type will be used to prove part (3) of Theorem III.2 and part (3) of Theorem I.8.

Some results of Ringel [8] and of Ringel and Youngs [9] are used repeatedly here, and so shall be stated separately as Theorem III.8.

Theorem III.8. (1) (Ringel [8]). Let $K_{2}=(2,4)$. Then for all $s \geq 0$ there is a triangular imbedding of $K_{12 s+5} \sim K_{2}$ in a surface of genus $g\left(K_{12 s+5}\right)-1$ such that there is a vertex 1 with adjacency tour 1) $2,3,4,5, \cdots, 12 s+5$.

(2) (Ringel and Youngs [9]). Let $K_{2}=(2,5)$. Then for all odd $s \geq 1$ there is a triangular imbedding of $K_{12 s+2}$ in a surface of genus $g\left(K_{12 s+2}\right)-1$ such that there is a vertex 1 with adjacency tour 1$) 2,3,4,5,6, \cdots, 12 s+2$.

In order to give some idea of how the se facts can be used and to reintroduce some of the ideas behind the proof of Proposition I.15, part (3) of Theorem III.2 will be considered first. It is now restated as Theorem III.9.

Theorem III.9. If one of $m$ and $n$ is congruent to 5 modulo 12 and the other is congruent to $1,2,5,6,8,9,10$, or 11 modulo 12 , then $g\left(K_{m} \vee K_{4} K_{n}\right)=g\left(K_{m}\right)+$ $g\left(K_{n}\right)-1$.

Note. As is shown by Table I.1, $m \equiv 1,2,5,6,8,9,10$, or $11(\bmod 12)$ if and only if $d\left(K_{m}\right) \neq 0$.

Proof. Let $K_{2}=(2,4)$ and applying Theorem III.8 find a triangular imbedding of $K_{12 s+5} \sim K_{2}$ in a surface of genus $g\left(K_{12 s+5}\right)-1$ with a vertex 1 whose adjacency tour is 1) $2,3,4, \cdots$. This means that the triangular faces $[2,1,3]$ and $[3,1,4]$ are adjacent, as shown in Figure 9.

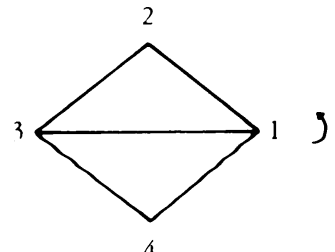

Figure; 
If the edge $(1,3)$ is deleted, the result is an imbedding of $K_{12 s+5} \sim\left\{K_{2}, K_{2}\right\}$ in a surface of genus $g\left(K_{12 s+5}\right)-1$ one face of which is $[1,4,3,2]$.

Moreover, as remarked above, if $m$ is congruent to $1,2,5,6,8,9,10$, or 11 modulo 12 , then $d\left(K_{m}\right) \neq 0$ and so there is a minimal imbedding of $K_{m}$ with a 4 sided face $\left[1^{\prime}, 2^{\prime}, 3^{\prime}, 4^{\prime}\right]$.

Now delete each of the above 4-sided faces from its respective surface and identify the boundaries in the obvious way, that is, identify each unprimed vertex with the primed vertex of the same number, and identify the corresponding edges. Note that this identification is made so as to preserve orientability.

Because $K_{m}$ is complete, the edges which were deleted from $K_{12 s+5}$ are restored in this construction. Hence one has obtained an imbedding of the amalgamation of $K_{m}$ and $K_{12 s+5}$ along $K_{4}$ in a surface of genus $g\left(K_{12 s+5}\right)+g\left(K_{m}\right)-1$. This completes the proof of the theorem, and hence the proof of Theorem III.2.

For convenience, Theorem I. 8 will now be restated as Theorem III.10.

Theorem III.10. (1) If $g\left(K_{m} \vee K_{5} K_{n}\right)=g\left(K_{m}\right)+g\left(K_{n}\right)-1$, then one of the following conditions is true.

(a) One of $m$ and $n$ is congruent to 2 or 5 modulo 12.

(b) One of $m$ and $n$ is congruent to 8 or 11 modulo 12 and the other is congruent to $1,6,8,9,10$, or 11 modulo 12 .

(2) If $K_{m}$ and $K_{n}$ are critical, then $g\left(K_{m} \vee_{K_{5}} K_{n}\right)=g\left(K_{m}\right)+g\left(K_{n}\right)-1$.

(3) If one of $m$ and $n$ is congruent to 5 modulo 12, then $g\left(K_{m} \vee_{K_{5}} K_{n}\right)=g\left(K_{m}\right)+$ $g\left(K_{n}\right)-1$.

As usual, the proof is by cases according to the value of $d\left(K_{m} \vee K_{s} K_{n}, g\left(K_{m}\right)+g\left(K_{n}\right)-1\right)$. These values are tabulated using the formula of Proposition I.11 and are displayed in Table III.2.

Table III. 2

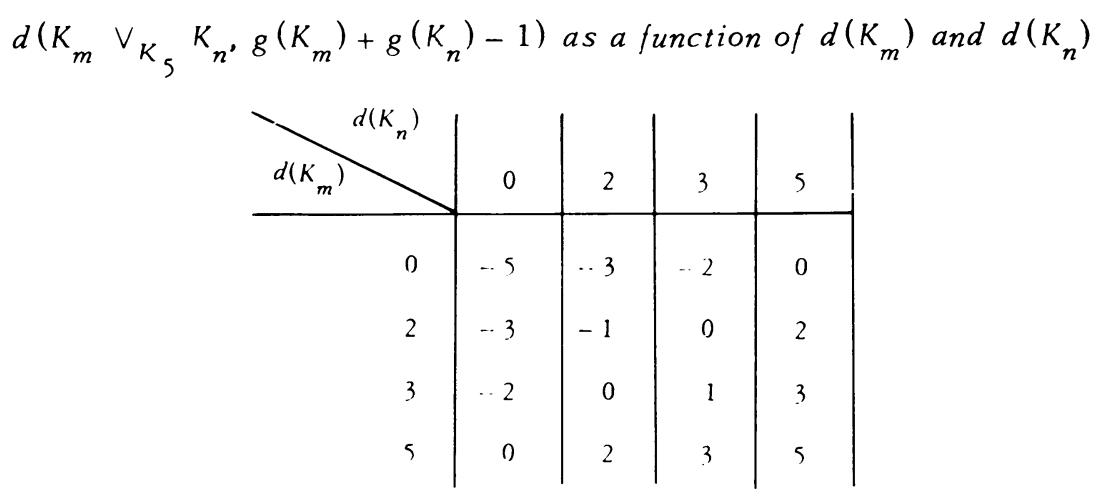

Note that (1) of Theorem III.10 simply summarizes the data of Table III.2 (see Table I.1). Part (2) of Theorem III.10 was proven as Corollary II.6. To prove 
(3), it suffices to show that $g\left(K_{m} \vee_{K_{5}} K_{n}\right) \leq g\left(K_{m}\right)+g\left(K_{n}\right)-1$ whenever one of $m$ and $n$ is congruent to 5 modulo 12. This follows from (1) of the next theorem.

Theorem III.11. (1) For all $s \geq 0$ and all $m, p \geq 5, g\left(K_{12 s+5} \vee K_{p} K_{m}\right) \leq$ $g\left(K_{12 s+5}\right)+g\left(K_{m}\right)-1$.

(2) For all odd $s \geq 1$ and all $m, p \geq 6, g\left(K_{12 s+2} \vee_{K_{p}} K_{m}\right) \leq g\left(K_{12 s+2}\right)+$ $g\left(K_{m}\right)-1$.

Proof. The proof is a slight modification of the proof of Proposition I.15. As it was shown there, one can always find a minimal imbedding of $K_{m}$ in a surface $M^{\prime}$ with a vertex $p^{\prime}$ with adjacency tour

$$
\left.p^{\prime}\right) m^{\prime},(m-1)^{\prime}, \ldots,(p+1)^{\prime},(p-1)^{\prime}, \ldots, 2^{\prime}, 1^{\prime}
$$

and such that for all $i$ with $1 \leq i \leq p-2$, the angle $(i+1)^{\prime} p^{\prime}{ }^{\prime}{ }^{\prime}$ is an angle of a triangular face. Delete all edges between $p^{\prime}$ and $2^{\prime}, 3^{\prime}, \cdots,(p-2)^{\prime}$ to obtain an imbedding of $K_{m} \sim K_{1}, p-3$ in $M^{\prime}$ with a $p$-sided face $P^{\prime}=\left[1^{\prime}, 2^{\prime}, \cdots, p^{\prime}\right]$.

In case (1), use Theorem III.8 (1) to find a triangular imbedding of $K_{12 s+5} \sim$ $K_{2}$ in a surface $M$ of genus $g\left(K_{12 s+5}\right)-1$ such that $K_{2}=(2,4)$ and the adjacency tour at 1 is 1) $2,3,4,5, \cdots, 12 s+5$.

The procedure now is identical to that used in the proof of Proposition I.15: delete the edges between 1 and $3,4, \cdots, p-1$ and remove the resulting $p$-sided face $[p, p-1, \cdots, 2,1]$ from $M$. Identify the boundary with that resulting from the removal of $P^{\prime}$ from $M^{\prime}$ in the obvious way. Note that the identification is made in such a way so as to preserve orientability.

Because $K_{m}$ and $K_{12 s+5}$ are complete, and since $p \geq 5$, it is clear that all the edges which were deleted from the two graphs are restored in this construction. Hence one has obtained, after deleting multiple edges, an imbedding of $K_{12 s+s}$ amalgamated with $K_{m}$ along $K_{p}$ in the connected sum of $M$ and $M^{\prime}$, that is, in a surface of genus $g\left(K_{m}\right)+g\left(K_{12 s+5}\right)-1$.

In case (2), Theorem III.8 (2) says that there is a triangular imbedding of $K_{12 s+2} \sim K_{2}, s$ odd, in a surface of genus $g\left(K_{12 s+2}\right)-1$ such that $K_{2}=(2,5)$ and the adjacency tour at 1 is 1) $2,3,4,5,6, \cdots, 12 s+2$.

Apparently, if one makes the added assumption that $p \geq 6$, exactly the same construction as was used to prove case (1) results in this case in an imbedding of $K_{12 s+2} V_{K_{p}} K_{m}$ in a surface of genus $g\left(K_{m}\right)+g\left(K_{12 s+2}\right)-1$. This completes the proof of the theorem.

Corollary III.12. Let $m=12 s+r$ and $n=12 t+q$, where $0 \leq r, q \leq 11$.

(1) If $r=5$ or $s$ is odd and $r=2$, and if $q \neq 2$ or 5 , then $g\left(K_{m} \vee K_{6} K_{n}\right)=$ $g\left(K_{n} \vee K_{6} K_{m}\right)=g\left(K_{m}\right)+g\left(K_{n}\right)-1$.

(2) If $r=5$ or $s$ is odd and $r=2$, and if $q=0,3,4$ or 7 , then $g\left(K_{m} \vee K_{7} K_{n}\right)=$ $g\left(K_{n} \vee_{K_{7}} K_{m}\right)=g\left(K_{m}\right)+g\left(K_{n}\right)-1$. 
Proof. By Theorem III.11, $g\left(K_{m} \vee_{K_{p}} K_{n}\right) \leq g\left(K_{m}\right)+g\left(K_{n}\right)-1$ in all of these cases. On the other hand, a computation using Proposition I.11 shows that $g\left(K_{m}\right)+g\left(K_{n}\right)-1$ is also a lower bound for $g\left(K_{m} \vee_{K_{p}} K_{n}\right)$ in these cases.

In some cases, the upper bound on $g\left(K_{m} \vee_{K_{p}} K_{n}\right)$ can actually be reduced to $g\left(K_{m}\right)+g\left(K_{n}\right)-2$.

Theorem III.13. Let $m=12 s+r$ and $n=12 t+q$, where $0 \leq r, q \leq 11$. Then $g\left(K_{m} \vee K_{p} K_{n}\right)=g\left(K_{n} \vee_{K_{p}} K_{m}\right) \leq g\left(K_{m}\right)+g\left(K_{n}\right)-2$ provided one of the following conditions is satisfied.

(1) $p \geq 6, r=5$ and either $q=5$ or else $t$ is odd and $q=2$.

(2) $p \geq 7, r=q=2$, and $s$ and $t$ are odd.

Proof. The proof involves only a slight modification of the proof of Theorem III.11, to which the reader is referred.

In case (1), one can replace the imbedding of $K_{m}$ used in the proof of Theorem III.11 with a triangular imbedding of $K_{12 s+5} \sim K_{2}$ in a surface of genus $g\left(K_{12 s+5}\right)-1$ such that $K_{2}=\left(3^{\prime}, 5^{\prime}\right)$ and the adjacency tour at $p^{\prime}$ is

$$
\left.p^{\prime}\right)(12 s+5)^{\prime}, \ldots,(p+1)^{\prime},(i-1)^{\prime}, \ldots, 6^{\prime}, 5^{\prime}, 4^{\prime}, 3^{\prime}, 2^{\prime}, 1^{\prime} .
$$

This is valid because $p \geq 6$.

If one now proceeds exactly as in the proof of Theorem III.11, one obtains the desired imbedding of $K_{m} \vee_{K_{p}} K_{n}$ in a surface of genus $g\left(K_{m}\right)+g\left(K_{n}\right)-2$.

In case (2), replace the imbedding of $K_{m}$ with a triangular imbedding of $K_{12 s+2} \sim K_{2}, s$ odd, in a surface of genus $g\left(K_{12 s+2}\right)-1$ such that $K_{2}=\left(3^{\prime}, 6^{\prime}\right)$ and the adjacency tour at $p^{\prime}$ is

$$
\left.p^{\prime}\right)(12 s+2)^{\prime}, \ldots,(p+1)^{\prime},(p-1)^{\prime}, \ldots, 7^{\prime}, 6^{\prime}, 5^{\prime}, 4^{\prime}, 3^{\prime}, 2^{\prime}, 1^{\prime} .
$$

This is valid because $p \geq 7$.

In this case, too, the procedure of Theorem III.11 yields the desired imbedding of $K_{m} \vee_{K_{p}} K_{n}$.

Corollary III.14. Let $m=12 s+r$ and $n=12 t+q, 0 \leq r, q \leq 11$.

(1) If $r=5$ and if $q=5$ or $q=2$ and $t$ is odd, then $g\left(K_{m} \vee_{K_{6}} K_{n}\right)=$ $g\left(K_{n} \vee K_{6} K_{m}\right)=g\left(K_{m}\right)+g\left(K_{n}\right)-2$.

(2) If $r=5$ or $s$ is odd and $r=2$, and if $q=5$ or $t$ is odd and $q=2$, then $g\left(K_{m} \vee_{K_{7}} K_{n}\right)=g\left(K_{n} \vee_{K_{7}} K_{m}\right)=g\left(K_{m}\right)+g\left(K_{n}\right)-2$.

Proof. By Theorem III.13, $g\left(K_{m}\right)+g\left(K_{n}\right)-2$ is an upper bound for $g\left(K_{m} \vee K_{p} K_{n}\right)$ in these cases. A computation using Proposition I.11 shows that it is also a lower bound. 


\section{SOME OPEN QUESTIONS}

A. General amalgamations along $K_{2}$. If arbitrary graphs $G$ and $H$ are amalgamated along an edge to form a $G \vee_{K_{2}} H$, then $g\left(G \vee_{K_{2}} H\right) \leq g(G)+g(H)$. Theorem II. 4 shows that under certain circumstances this upper bound can be improved to $g(G)+g(H)-1$. No examples have been found for which the genus of $G \vee_{K_{2}} H$ is less than $g(G)+g(H)-1$.

These facts lead one to pose the following two interesting questions.

(1) Is $g\left(G \vee_{K_{2}} H\right) \geq g(G)+g(H)-1$ for all graphs $G$ and $H$ ?

(2) Is the converse of Theorem II.4 true? Recall that Theorem II.3 gives an affirmative answer to (2) in a special case.

B. Monotonicity. If $p$ and $q$ are integers $\leq 5$, and if $p \leq q$, then the results of this paper imply that for all $m$ and $n \geq q, g\left(K_{m} \vee K_{p} K_{n}\right) \geq g\left(K_{m} \vee_{K_{q}} K_{n}\right)$. It is natural to ask whether this inequality holds in general.

Some caution is in order here, as the following example will show. For arbitrary graphs $G$ and $H$ and subgraphs $T \subseteq T^{\prime}$, the corresponding question is whether or not $g\left(G \vee_{T} H\right) \geq g\left(G \vee_{T}, H\right)$. It is easy to construct an example for which this is not true. In fact, if $G, H, T$, and $T^{\prime}$ are as in Figure 10, then clearly $T \subseteq T^{\prime}$. However, $G \vee_{T} H$ is an amalgamation of $K_{5}$ and a planar graph along $K_{1}$, and so $g\left(G \vee_{T} H\right)=1$, while $G \vee_{T}, H=K_{S} \vee_{K_{1}} K_{5}$, and hence $g\left(G \vee_{T}, H\right)=2$.

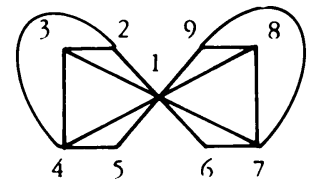

(i

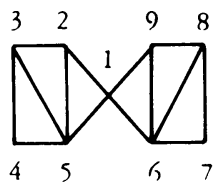

H

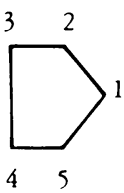

$T$

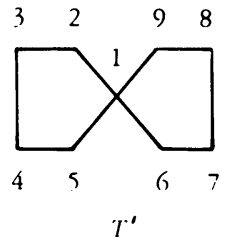

$T^{\prime \prime}$

Figure 10

For complete graphs this question amounts to asking whether for all $m, n$, and $p$ such that $m, n \geq p+1$ the following inequality holds:

$$
g\left(K_{m} \vee_{K_{p}} K_{n}\right) \geq g\left(K_{m} \vee_{K_{p+1}} K_{n}\right) .
$$

Note that if $p$ is close to $m$ and $n$ the question has an affirmative answer. For example, if $m \geq 12$, then

$$
\begin{aligned}
& \quad g\left(K_{m} \vee_{K_{m}} K_{m+1}\right)=g\left(K_{m+1}\right), \\
& g\left(K_{m} \vee_{K_{m-1}} K_{m+1}\right)=g\left(K_{m+2} \sim K_{1,2}\right) \geq g\left(K_{m+2}\right)-1>g\left(K_{m+1}\right), \\
& g\left(K_{m} \vee_{K_{m-2}} K_{m+1}\right)=g\left(K_{m+3} \sim K_{2,3}\right) \geq g\left(K_{m+3}\right)-1>g\left(K_{m+2}\right) .
\end{aligned}
$$


The strict inequalities are consequences of the Complete Graph Theorem and the fact that $m \geq 12$. The definition of triangulation deficiency is such that for graphs $G$ and nonnegative integers $g, d(G, g)=d(G, g-1)+6$. Furthermore, if $H$ has $E(H)$ edges, then $d(G \sim H, g)=d(G, g)-E(H)$. Hence the inequalities $g\left(K_{m+2} \sim K_{1,2}\right) \geq g\left(K_{m+2}\right)-1$ and $g\left(K_{m+3} \sim K_{2,3}\right) \geq g\left(K_{m+3}\right)-1$ follow from Proposition I.9 (3) and the fact that for all $m, d\left(K_{m}\right) \leq 5$.

C. Improved upper bounds. The apparent difficulty of obtaining a better upper bound than that given by Proposition I.15 for $g\left(K_{m} \vee_{K_{p}} K_{n}\right)$ when $p$ is large has already been commented upon. What seems to be missing is something accounting for $g\left(K_{p}\right)$. It is a consequence of Proposition I.11 that

$$
\Delta=g\left(K_{m}\right)+g\left(K_{n}\right)-g\left(K_{p}\right)+\left\{\left(d\left(K_{p}\right)-d\left(K_{m}\right)-d\left(K_{n}\right)\right) / 6\right\} \leq g\left(K_{m} \vee_{K_{p}} K_{n}\right) .
$$

This lower bound is not always exact. However, in all cases for which $g\left(K_{m} \vee K_{p} K_{n}\right)$ is actually computed in this thesis one is able to show (cf. Theorem I.4) that $\Delta \leq g\left(K_{m} \vee_{K_{p}} K_{n}\right) \leq \Delta+1$.

One would like to have something like this for all $p$. Note that this would help answer the monotonicity question of $\S$ IV.B.

D. Is $K_{24 s+2}$ critical? This question has been a persistent source of frustration in this paper, due to the unresolved cases involving graphs of the form $K_{24 s+2}$. Most of these cases would be resolved if it could be shown that $K_{24 s+2}, s \geq 1$, was critical. The apparent difficulty of this question has already been noted by Ringel and Youngs [12].

Note, however, that it seems even harder to show that $K_{24 s+2}$ is not critical, for one must then show that none of the 2-cell imbeddings of $K_{24 s+2} \sim K_{2}$ is triangular.

Even if $K_{24 s+2}$ is not critical, it may still have a minimal imbedding with a 7 -sided face with 2 repeated vertices. Thus one may still be able to apply Theorem II.4 to amalgamations of other complete graphs with $K_{24 s+2}$.

These facts lead one to believe that a determination in all cases of $g\left(K_{m} \vee_{K_{p}} K_{n}\right), p \leq 5$, is not close at hand.

\section{REFERENCES}

1. J. Battle, F. Harary, Y. Kodama and J. W. T. Youngs, Additivity of the genus of a graph, Bull. Amer. Math. Soc. 68 (1962), 565-568. MR 27 \#5247.

2. J. Ch. Boland, Embedding of graphs into orientable surfaces, Nederl. Akad. Wetensch. Proc. Ser. A 70 = Indag. Math. 29 (1967), 33-44. MR 35 \#2272.

3. J. Edmonds, A combinatorial representation for polyhedral surfaces, Notices Amer. Math. Soc. 7 (1960), 646. Abstract \#572-1. 
4. W. Gustin, Orientable imbedding of Cayley graphs, Bull. Amer. Math. Soc..69 (1963), 272-275. MR $26 \# 3037$.

5. F. Harary, Graph theory, Addison-Wesley, Reading, Mass., 1969. MR 41 \#1566.

6. I. N. Kagno, The mapping of graphs on surfaces, J. Math. and Phys. 16 (1937), 46-75.

7. J. Mayer, Le problème des régions voisines sur les surfaces closes orientables, J. Combinatorial Theory 6 (1969), 177-195. MR 38 \#3177.

8. G. Ringel, Färbungsprobleme auf Flächen und Graphen, Mathematische Monographien, 2, VEB Deutscher Verlag der Wissenschaften, Berlin, 1959. MR 22 \#235.

9. G. Ringel and J. W. T. Youngs, Lösung des Problems der Nachbargebiete, Arch. Math. (Basel) 20 (1969), 190-201. MR 39 \#4049.

10. Solution of the Heawood map-coloring problem, Proc. Nat. Acad. Sci. U. S. A. 60 (1968), 438-445. MR 37 \#3959.

11. - Solution of the Heawood map-coloring problem-case 11, J. Combinatorial Theory 7 (1969), 71-93. MR 39 \#1360.

12. - Solution of the Heawood map-coloring problem-case 2, J. Combinatorial Theory 7 (1969), 342-352. MR 42 \#128.

13. - Solution of the Heawood map-coloring problem-case 8, J. Combinatorial Theory 7 (1969), 353-363. MR 41 \#6723.

14. C. M. Terry, L. R. Welch and J. W. T. Youngs, The genus of $K_{12 s}$, J. Combinatorial Theory 2 (1967), 43-60. MR 34 \#6755.

15. - Solution of the Heawood map-coloring problem-case 4, J. Combinatorial Theory 8 (1970), 170-174. MR $41 \# 3321$.

16. J. W. T. Youngs, The Heawood map coloring conjecture, Graph Theory and Theoretical Physics, Academic Press, London, 1967, pp. 313-354. MR 38 \#357.

17. - Minimal imbeddings and the genus of a graph, J. Math. Mech. 12 (1963), 303-315. MR $26 \# 3043$.

18. J. W. T. Youngs, Solution of the Heawood map-coloring problem-cases 3, 5, 6, and 9, J. Combinatorial Theory 8 (1970), 175-219. MR $41 \# 3322$.

19. - Solution of the Heawood map-coloring problem-cases 1, 7, and 10, J. Combinatorial Theory 8 (1970), 220-231. MR $41 \# 3323$.

DEPARTMENT OF MATHEMATICS, COLUMBIA UNIVERSITY, NEW YORK, NEW YORK 10027

Current address: Downstate Medical Center, Department of Medical Computer Science, Box \#7, Brooklyn, New York 11203 